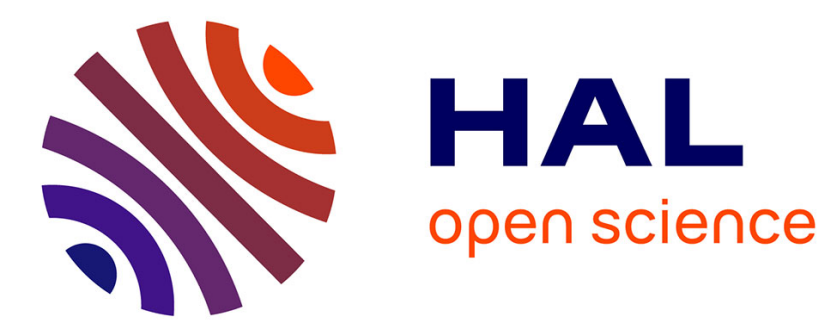

\title{
Optimized weak coupling of boundary element and finite element methods for acoustic scattering
}

Boris Caudron, Xavier Antoine, Christophe Geuzaine

\section{To cite this version:}

Boris Caudron, Xavier Antoine, Christophe Geuzaine. Optimized weak coupling of boundary element and finite element methods for acoustic scattering. Journal of Computational Physics, 2020, 421, 10.1016/j.jcp.2020.109737 . hal-02524580

\section{HAL Id: hal-02524580 \\ https://hal.science/hal-02524580}

Submitted on 30 Mar 2020

HAL is a multi-disciplinary open access archive for the deposit and dissemination of scientific research documents, whether they are published or not. The documents may come from teaching and research institutions in France or abroad, or from public or private research centers.
L'archive ouverte pluridisciplinaire HAL, est destinée au dépôt et à la diffusion de documents scientifiques de niveau recherche, publiés ou non, émanant des établissements d'enseignement et de recherche français ou étrangers, des laboratoires publics ou privés. 


\title{
Optimized weak coupling of boundary element and finite element methods for acoustic scattering
}

\author{
B. Caudron ${ }^{1,2,3}$, X. Antoine ${ }^{1}$ and C. Geuzaine ${ }^{2}$
}

\begin{abstract}
In this paper we present an optimized weak coupling of boundary element and finite element methods to solve acoustic scattering problems. This weak coupling is formulated as a nonoverlapping Schwarz domain decomposition method, where the transmission conditions are constructed through Padé localized approximations of the Dirichlet-to-Neumann map. The performance of the resulting formulations is analyzed on several three-dimensional examples, with both homogeneous and inhomogeneous scatterers.
\end{abstract}

\section{Introduction}

Acoustic scattering problems in the frequency domain have multiple applications in science, engineering and medicine, from sonar to ultrasound imaging. Fundamentally, the numerical solution of such problems is based on the discretization of the three-dimensional Helmholtz equation in an unbounded homogeneous domain, but where the scatterer can be inhomogeneous, i.e. with spatially-varying density and wave speed. The important characteristic to be computed is often the far field response, e.g. through the calculation of the Sonar Cross Section (SCS) that encapsulates the acoustic response of the scatterer.

Solving the Helmholtz equation in such configurations at high frequencies (when the wavelength is small compared to the size of the scatterer) is a challenging problem. A standard approach is to truncate the computational domain using Artificial Boundary Conditions [1, 4, 20] or Perfectly Matched Layers [6, 8, 38, and to use e.g. the Finite Element Method (FEM) to discretize the Helmholtz equation in the resulting bounded domain. The main advantage of FEM is its flexibility in dealing with complex media, in particular for heterogeneous scatterers. Another approach is to couple FEM with a surface integral equation set on the boundary of the scatterer [32], discretized e.g. using the Boundary Element Method (BEM). The main advantage of BEM is its ability to accurately deal with the unboundedness of the domain: the integral formulation provides an exact representation of the exterior scattered field (through the Green's function) unlike ABCs

\footnotetext{
${ }^{1}$ Université de Lorraine, CNRS, Inria, IECL, F-54000 Nancy, France.

Email: xavier.antoine@univ-lorraine.fr

${ }^{2}$ Université de Liège, Institut Montefiore B28, B-4000 Liège, Belgium. Email: cgeuzaine@uliege.be

${ }^{3}$ Thales, 2 Avenue Gay-Lussac, 78851 Elancourt cedex, France.
} 
and PMLs. Moreover, advances in compression techniques like Hierarchical Matrices (HMatrices) [9, 25] or Fast Multipole Methods (FMM) [24] allow to alleviate the fact that BEM naturally leads to dense matrices, whereas FEM yields sparse matrices. FEM-BEM coupling [5, 11, 12, 15, 16, 23, 27, 29, 33, 39, thus combines the advantages of both methods to solve complex acoustic scattering problems.

Classically, FEM-BEM coupling is constructed by coupling the variational volume formulation and the surface integral formulation within a single equation [12, 15, 16, 23, 27, 29, 33, 39, leading to a so-called strong FEM-BEM coupling. While natural and easy to set up, this approach however exhibits several drawbacks. First, the resulting system combines dense and sparse blocks and is not directly amenable to efficient compression through H-Matrices and FMM. Moreover, no robust preconditioner exists for iterative linear solvers for the FEM-BEM coupling at high-frequencies [21, 28]. Finally, if two existing optimized solvers for FEM and BEM already exist, implementing an efficient strong FEM-BEM coupling is non trivial - or downright impossible.

As an alternative to strong FEM-BEM coupling, other FEM-BEM couplings have been proposed [5, 11, which correspond concretely to Schwarz domain decomposition methods iterating between the two domains, i.e. the scatterer and the outside domain. Conceptually, constructing the FEM-BEM coupling as a domain decomposition method presents a major advantage: it allows to only weakly couple the volume variational formulation and the surface integral equation, each using e.g. an optimized code. From an industrial perspective, using two independent solvers can also be very interesting: different partners can exchange only interface data without sharing the underlying detailed models for each subproblem.

The robustness and the convergence rate of domain decomposition methods strongly depends on the transmission conditions between the subdomains [17], the optimal transmission operators being, for each subdomain, the Dirichlet-to-Neumann (DtN) maps corresponding to the complementary subdomains [18]. The DtN operator being a nonlocal operator, approximate local versions are rather used in practice. Recently, new accurate approximations of the DtN map have been proposed [2, 10, based on the Padé approximation of a square-root operator. In the context of domain decomposition methods for FEM, these new conditions led to a convergence rate that is almost independent of the frequency and mesh size. In this paper, we derive optimized weak FEM-BEM coupling formulations based on these transmission boundary conditions. We also propose a BEM-BEM coupling, which can be advantageously used when the scatterer is homogeneous.

The paper is organized as follows. In Section 2, we define the transmission scattering boundary-value problem under study. Section 3 is devoted to the derivation of the weak coupling approach, while the formulations for the subproblems are developed in Section 4. The Padé approximation of the DtN map is detailed in Section 5. The performance of the resulting optimized weak BEM-BEM and FEM-BEM coupling formulations is analyzed on several numerical examples in Section 6. Finally, we conclude in Section 7 . 


\section{The transmission scattering boundary-value problem}

Let us consider $\Omega_{-}$as a three-dimensional bounded obstacle of $\mathbb{R}^{3}$, with boundary $\Gamma:=$ $\partial \Omega_{-}$, and $\Omega_{+}:=\mathbb{R}^{3} \backslash \overline{\Omega_{-}}$as the associated exterior domain of propagation. The vector $\mathbf{n}$ designates the outwardly directed unit normal vector to $\Omega_{-}$. We consider an incident pressure wave, denoted by $u_{\text {inc }}$, travelling into the unbounded domain $\Omega_{+}$, and satisfying the exterior Helmholtz equation

$$
\Delta u_{\text {inc }}+k_{+}^{2} u_{\text {inc }}=0 \text { in } \Omega_{+} .
$$

The wave number $k_{+}$is real-valued assuming that $\Omega_{+}$is homogeneous and non dissipative, the incident wave field being a plane wave: $u_{\text {inc }}=e^{-i \mathbf{k}_{+} \cdot \mathbf{x}}$, with $\mathbf{k}_{+}=k_{+} \boldsymbol{\sigma}_{\text {inc }}$ and $\left\|\boldsymbol{\sigma}_{\text {inc }}\right\|=1$. Hereabove, the inner hermitian product is defined by $\mathbf{a} \cdot \mathbf{b}$ and the associated norm is $\|\mathbf{a}\|$, for two complex-valued vectors $\mathbf{a}$ and $\mathbf{b}$ in $\mathbb{C}^{3}$.

Let us now introduce the transmission problem that we want to solve: find the acoustic field $u$ solution to

$$
\begin{aligned}
& \operatorname{div}(\rho \boldsymbol{\nabla} u)+\rho k_{-}^{2} u=0 \text { in } \Omega_{-}, \\
& \Delta u+k_{+}^{2} u=0 \text { in } \Omega_{+}, \\
& \partial_{r} u-i k_{+} u=\underset{r \rightarrow+\infty}{\mathcal{O}}\left(r^{-2}\right), \\
& \gamma_{D}^{-} u=\gamma_{D}^{+} u+\gamma_{D}^{+} u_{\text {inc }} \text { on } \Gamma, \\
& \gamma_{n}^{-}(\rho \nabla u)=\gamma_{N}^{+} u+\gamma_{N}^{+} u_{\text {inc }} \text { on } \Gamma,
\end{aligned}
$$

where the relative density $\rho$ is defined as $\rho=\rho_{+} / \rho_{-}$, with $\rho_{ \pm}$the density in the domain $\Omega_{ \pm}$, and where the interior wave number $k_{-}$in $\Omega_{-}$, as well as $\rho_{-}$, can be space-dependent functions of $\mathbf{x}$ to account for inhomogeneous materials in $\Omega_{-}$. The operators div and $\nabla$ denote the divergence and gradient operators, respectively, and the Laplacian is defined by $\Delta:=\operatorname{div} \boldsymbol{\nabla}$. The first equation of the system is called the Bergmann equation [7] while the second one is the exterior Helmholtz equation. The third equation is the Sommerfeld radiation condition at infinity, which also writes

$$
\lim _{r \rightarrow \infty} r\left(\partial_{r} u-i k_{+} u\right)=0,
$$

where $r$ is the radial variable. The two last equations, called continuity equations, involve the Dirichlet and Neumann trace operators, i.e. $\gamma_{D}^{ \pm}$and $\gamma_{N}^{ \pm}$, for the domains $\Omega^{ \pm}$. In addition, we define $\gamma_{n}^{ \pm} \mathbf{u}=\mathbf{u}_{\mid \Gamma} \cdot \mathbf{n}$, for a smooth enough vector field $\mathbf{u} \in \mathbb{C}^{3}$.

\section{The weak coupling approach}

When solving the transmission problem (1), a system of two coupled equations has to be written equivalently and is then numerically discretized. These formulations involve integral or/and volume equations, which are then discretized thanks to boundary (BEM) or/and finite element methods (FEM) according to the situation. In this way a linear system 
of two equations with two unknowns has to be solved: this coupling between the interior and exterior problems is usually referred to as strong coupling.

We propose here an alternative approach which consists in decoupling the two equations through an iterative process that exchanges suitable information at the interface $\Gamma$ between the interior and exterior domains. Therefore, our point of view is related to a domain decomposition method without overlap, with two subdomains [37]. For Helmholtz-type problems, it is well-known that considering a decomposition related to the continuity boundary conditions $(1 \mathrm{~d})-1 \mathrm{e})$ can lead to a divergent algorithm, as an eigenvector of the Laplace operator can exist in the interior subdomain which satisfies the boundary conditions [30]. A solution to get a convergent iterative method is to write a Schwarz domain decomposition method between the interior and exterior domains of (1) using transmission conditions combining Dirichlet and Neumann data [30]. In their simplest form, the transmission conditions are of Fourier-Robin type with constant coefficients [17]. More advanced conditions, related to approximations of the Dirichlet-to-Neumann (DtN) operators for (1), lead to improved convergence properties [10]-which is crucial in the context of this work as aim to minimizing of the number of calls to the resolution procedure for the interior and exterior problems. The resulting methods are called optimized Schwarz non-overlapping domain decomposition methods [10, 22].

\subsection{Transmission and resolution operators}

To reformulate the boundary conditions $(1 \mathrm{~d})-(1 \mathrm{e})$, let us introduce two transmission operators $T_{ \pm}: \mathrm{H}^{\frac{1}{2}}(\Gamma) \rightarrow \mathrm{H}^{-\frac{1}{2}}(\Gamma)$. From now on, we assume that $T_{-}-T_{+}$is injective. The transmission conditions (1d)-(1e) are then equivalent to the following transmission boundary conditions

$$
\left\{\begin{array}{l}
\gamma_{n}^{-}(\rho \nabla u)+T_{-} \gamma_{D}^{-} u=\gamma_{N}^{+} u+T_{-} \gamma_{D}^{+} u+\gamma_{N}^{+} u_{\mathrm{inc}}+T_{-} \gamma_{D}^{+} u_{\mathrm{inc}} \\
\gamma_{N}^{+} u+T_{+} \gamma_{D}^{+} u=\gamma_{n}^{-}(\rho \boldsymbol{\nabla} u)+T_{+} \gamma_{D}^{-} u-\gamma_{N}^{+} u_{\mathrm{inc}}-T_{+} \gamma_{D}^{+} u_{\mathrm{inc}}
\end{array}\right.
$$

Let us remark that if the transmission boundary conditions $(1 \mathrm{~d})-(1 \mathrm{e})$ are satisfied, then this implies that we also have (2). Conversely, the injectivity of $T_{-}-T_{+}$proves the equivalence. Usual domain decomposition methods assume that $T_{+}=-T_{-}$(see e.g. [5, 10, 17]). Here, we nevertheless consider that the two operators may be different to get a more general framework but also to gain in flexibility. As previously mentioned, rewriting the initial problem (1) through well-adapted transmission boundary conditions is required to design a converging domain decomposition method. In particular, the conditioning of the method is directly impacted by the choice of the transmission operators. In this paper, we use an iterative Krylov subspace method (GMRES) [34].

To obtain the new formulation of the diffraction-transmission problem (1), we need a few definitions. First, we introduce the two following trace operators

$$
B_{-}=\gamma_{n}^{-}(\rho \nabla)+T_{-} \gamma_{D}^{-}, \quad B_{+}=\gamma_{N}^{+}+T_{+} \gamma_{D}^{+},
$$

from which we define the new surface fields $g_{ \pm}=B_{ \pm} u$. Even if this will only appear later, the domain decomposition method allows to interpret $g_{-}$and $g_{+}$as some transmitted quantities from $\Omega_{+}$to $\Omega_{-}$and from $\Omega_{-}$to $\Omega_{+}$. Since we reformulate the diffraction-transmission 
problem (1), we also need to introduce some resolution operators, denoted by $R_{-}$and $R_{+}$, and defined by: $\forall g \in \mathrm{H}^{-\frac{1}{2}}(\Gamma), R_{ \pm} g=\gamma_{D}^{ \pm} u_{ \pm}$, the functions $u_{-} \in \mathrm{H}^{1}\left(\Omega_{-}\right)$and $u_{+} \in \mathrm{H}_{\mathrm{loc}}^{1}\left(\overline{\Omega_{+}}\right)$ being the solutions to the respective boundary-value problems

$$
\operatorname{div}\left(\rho \nabla u_{-}\right)+\rho k_{-}^{2} u_{-}=0 \text { in } \Omega_{-}, \quad B_{-} u_{-}=g,
$$

and

$$
\Delta u_{+}+k_{+}^{2} u_{+}=0 \text { in } \Omega_{+}, \quad B_{+} u_{+}=g, \quad \partial_{r} u_{+}-i k_{+} u_{+}=\mathcal{O}\left(\frac{1}{r^{2}}\right) .
$$

In practice, the resolution operators are the continuous versions of the corresponding preexisting solvers related to the interior and exterior problems. They are well-defined if the problems (3) and (4) are well-posed. This clearly depends on the choice of the transmission operators. From now on, we assume that this is indeed the case. Let us remark that (3) and (4) correspond to the subproblems associated to the domain decomposition method for the weak coupling.

\subsection{Weak coupling formulation}

We can now introduce the weak coupling formulation of (1).

Proposition 3.1. If $u$ is solution to the diffraction-transmission problem (1), then $g_{-}$and $g_{+}$are solutions to

$$
\left(I d-S_{\pi}\right)\left(\begin{array}{c}
g_{-} \\
g_{+}
\end{array}\right)=\left(\begin{array}{c}
\gamma_{N}^{+} u_{\mathrm{inc}}+T_{-} \gamma_{D}^{+} u_{\mathrm{inc}} \\
-\gamma_{N}^{+} u_{\mathrm{inc}}-T_{+} \gamma_{D}^{+} u_{\mathrm{inc}}
\end{array}\right),
$$

the operator $S_{\pi}$ being defined from $S_{-}$and $S_{+}$by

$$
S_{\pi}=\left(\begin{array}{cc}
0 & S_{+} \\
S_{-} & 0
\end{array}\right), \quad S_{-}=I d-\left(T_{-}-T_{+}\right) R_{-}, \quad S_{+}=I d+\left(T_{-}-T_{+}\right) R_{+} .
$$

Proof The new transmission conditions (2) can be rewritten as

$$
\begin{aligned}
& B_{-} u=B_{+} u+\left(T_{-}-T_{+}\right) \gamma_{D}^{+} u+\gamma_{N}^{+} u_{\mathrm{inc}}+T_{-} \gamma_{D}^{+} u_{\mathrm{inc}}, \\
& B_{+} u=B_{-} u-\left(T_{-}-T_{+}\right) \gamma_{D}^{-} u-\gamma_{N}^{+} u_{\mathrm{inc}}-T_{+} \gamma_{D}^{+} u_{\mathrm{inc}} .
\end{aligned}
$$

Since $u$ is solution to

$$
\operatorname{div}(\rho \boldsymbol{\nabla} u)+\rho k_{-}^{2} u=0 \text { in } \Omega_{-}, \quad B_{-} u=g_{-},
$$

and

$$
\Delta u+k_{+}^{2} u=0 \text { in } \Omega_{+}, \quad B_{+} u=g_{+}, \quad \partial_{r} u-i k_{+} u=\mathcal{O}\left(\frac{1}{r^{2}}\right),
$$

we deduce that: $\gamma_{D}^{ \pm} u=R_{ \pm} g_{ \pm}$. Then, one gets

$$
\begin{aligned}
& g_{-}=g_{+}+\left(T_{-}-T_{+}\right) R_{+} g_{+}+\gamma_{N}^{+} u_{\mathrm{inc}}+T_{-} \gamma_{D}^{+} u_{\mathrm{inc}} \\
& g_{+}=g_{-}-\left(T_{-}-T_{+}\right) R_{-} g_{-}-\gamma_{N}^{+} u_{\mathrm{inc}}-T_{+} \gamma_{D}^{+} u_{\mathrm{inc}} .
\end{aligned}
$$

Finally, it is sufficient to check that the new transmission boundary conditions (2) are satisfied, which can be easily proved. 
As said before, a GMRES solver will be applied to the weak coupling formulation (5). Clearly, the rate of convergence of the iterative method strongly depends on the choice of the transmission operators. Even worse, a bad choice of the transmission operator could yield a divergence of the algorithm. We propose here some optimal transmission operators leading to a trivial resolution of the weak coupling formulation (5). This provides a guideline for designing well-adapted transmission operators for practical computations. Concretely, the optimal transmission operators correspond to DtN operators.

Let us introduce the interior (respectively exterior) DtN operator $\Lambda_{-, k, \sigma}$ (respectively $\Lambda_{+, k}$ ) associated to the problem (3) (respectively (4)). We then have

$$
\left(\Lambda_{-, k_{-}, \rho}+T_{-}\right) R_{-}=I d, \quad\left(\Lambda_{+, k_{+}}+T_{+}\right) R_{+}=I d,
$$

leading to

$$
\begin{array}{lll}
T_{-}=-\Lambda_{+, k_{+}} & \Rightarrow & S_{+}=0, \\
T_{+}=-\Lambda_{-, k_{-}, \rho} & \Rightarrow & S_{-}=0 .
\end{array}
$$

This result is particularly interesting since suitably choosing one of the transmission operators $T_{ \pm}$implies that $S_{\pi}$ is null, leading to a trivial resolution of the weak coupling (5). In fact, this result is even deeper. Indeed, let us assume that the transmission operators are such that $S_{-}=0$ or $S_{+}=0$. Then the operator $S_{\pi}$ is non null. Nevertheless, it is nilpotent and its spectrum is reduced to $\{0\}$. As a consequence, $I d-S_{\pi}$ is a priori well-adapted to a GMRES procedure since its eigenvalues cluster around $(1,0)$ in the complex plane, providing then a fast convergence rate of the method.

\section{Formulations for solving the subproblems}

We now present the formulations used for solving both the interior (3) and exterior (4) problems. We introduce the general formulations without specifying yet the transmission operators. This generic approach allows us to state a few theoretical results concerning the related formulations. The transmission operators used in practice as well as the associated formulations are presented in section 5 .

\subsection{Basic results about integral representations for the Helmholtz equation}

For $k \in \mathbb{R}_{+}^{*}$, we denote by $G_{k}$ the three-dimensional Green's function defined by

$$
\forall \mathbf{x} \in \mathbb{R}^{3} \backslash\{\mathbf{0}\}, \quad G_{k}(\mathbf{x})=\frac{e^{i k\|\mathbf{x}\|}}{4 \pi\|\mathbf{x}\|} .
$$

Let us introduce the surface single- $\left(S_{k}\right)$ and double-layer $\left(D_{k}\right)$ potentials, the adjoint doublelayer $\left(D_{k}^{*}\right)$ operator and the hypersingular $\left(N_{k}\right)$ operator, as continuously defined between the corresponding spaces following

$$
\begin{array}{rclccccc}
S_{k}: \mathrm{H}^{-\frac{1}{2}}(\Gamma) & \rightarrow & \mathrm{H}^{\frac{1}{2}}(\Gamma), & D_{k}: & \mathrm{H}^{\frac{1}{2}}(\Gamma) & \rightarrow & \mathrm{H}^{\frac{1}{2}}(\Gamma) \\
p & \mapsto & S_{k} p & & p & \mapsto & D_{k} p \\
D_{k}^{*}: \mathrm{H}^{-\frac{1}{2}}(\Gamma) & \rightarrow & \mathrm{H}^{-\frac{1}{2}}(\Gamma) & N_{k}: & \mathrm{H}^{\frac{1}{2}}(\Gamma) & \rightarrow & \mathrm{H}^{-\frac{1}{2}}(\Gamma) \\
p & \mapsto & D_{k}^{*} p & & p & \mapsto & N_{k} p
\end{array}
$$


where $\forall \mathbf{x} \in \Gamma$. We have

$$
\begin{array}{rrr}
S_{k} p(\mathbf{x})=\int_{\Gamma} G_{k}(\mathbf{x}-\mathbf{y}) p(\mathbf{y}) d \mathbf{y}, & D_{k} p(\mathbf{x})=\int_{\Gamma} \partial_{\mathbf{n}(\mathbf{y})} G_{k}(\mathbf{x}-\mathbf{y}) p(\mathbf{y}) d \mathbf{y} \\
D_{k}^{*} p(\mathbf{x})=\int_{\Gamma} \partial_{\mathbf{n}(\mathbf{x})} G_{k}(\mathbf{x}-\mathbf{y}) p(\mathbf{y}) d \mathbf{y}, & N_{k} p(\mathbf{x})=\int_{\Gamma} \partial_{\mathbf{n}(\mathbf{x})} \partial_{\mathbf{n}(\mathbf{y})} G_{k}(\mathbf{x}-\mathbf{y}) p(\mathbf{y}) d \mathbf{y} .
\end{array}
$$

Trace relations [14] can be obtained for these integral operators over $\Gamma$. Finally, setting $\left[\gamma_{D} u\right]=\gamma_{D}^{-} u-\gamma_{D}^{+} u$ and $\left[\gamma_{N} u\right]=\gamma_{N}^{-} u-\gamma_{N}^{+} u$, we have

$$
\forall \mathbf{x} \in \Omega_{-} \cup \Omega_{+}, \quad u(\mathbf{x})=\int_{\Gamma} G_{k}(\mathbf{x}-\mathbf{y})\left[\gamma_{N} u\right](\mathbf{y})-\partial_{\boldsymbol{n}(\mathbf{y})} G_{k}(\mathbf{x}-\mathbf{y})\left[\gamma_{D} u\right](\mathbf{y}) d \mathbf{y} .
$$

\subsection{Exterior problem formulations}

Let us start by introducing an equivalent integral equation formulation for the exterior problem (4). This formulation corresponds to a generalization of the one proposed in [3] for the electromagnetic scattering problem with impedance boundary condition.

Proposition 4.1. Let $g \in \mathrm{H}^{-\frac{1}{2}}(\Gamma)$ and $u \in \mathrm{H}_{\mathrm{loc}}^{1}\left(\overline{\Omega_{+}}\right)$such that:

$$
\Delta u+k_{+}^{2} u=0 \text { in } \Omega_{+} \quad, \quad \gamma_{N}^{+} u+T_{+} \gamma_{D}^{+} u=g \quad, \quad \partial_{r} u-i k_{+} u=\mathcal{O}\left(\frac{1}{r^{2}}\right) .
$$

We then have

$$
\frac{1}{2} T_{+}^{-1} \gamma_{N}^{+} u+D_{k_{+}} \gamma_{D}^{+} u-S_{k_{+}} \gamma_{N}^{+} u=\frac{1}{2} T_{+}^{-1} g, \quad \frac{1}{2} T_{+} \gamma_{D}^{+} u-D_{k_{+}}^{*} \gamma_{N}^{+} u+N_{k_{+}} \gamma_{D}^{+} u=\frac{1}{2} g .
$$

Conversely, if $g \in \mathrm{H}^{-\frac{1}{2}}(\Gamma), p \in \mathrm{H}^{\frac{1}{2}}(\Gamma)$ and $q \in \mathrm{H}^{-\frac{1}{2}}(\Gamma)$ are such that

$$
\frac{1}{2} T_{+}^{-1} q+D_{k_{+}} p-S_{k_{+}} q=\frac{1}{2} T_{+}^{-1} g, \quad \frac{1}{2} T_{+} p-D_{k_{+}}^{*} q+N_{k_{+}} p=\frac{1}{2} g,
$$

then the function $u \in \mathrm{H}_{\mathrm{loc}}^{1}\left(\overline{\Omega_{+}}\right)$defined by $u=-\mathcal{S}_{k_{+}} q+\mathcal{D}_{k_{+}}$in $\Omega_{+}$satisfies

$$
\Delta u+k_{+}^{2} u=0 \text { in } \Omega_{+}, \quad \gamma_{N}^{+} u+T_{+} \gamma_{D}^{+} u=g, \quad \partial_{r} u-i k_{+} u=\mathcal{O}\left(\frac{1}{r^{2}}\right) .
$$

Proof Let $u$ be the solution to the exterior problem (4). Extending $u$ by 0 in $\Omega_{-}$, one gets the following integral representation formula

$$
u=-\mathcal{S}_{k_{+}} \gamma_{N}^{+} u+\mathcal{D}_{k_{+}} \gamma_{D}^{+} u \text { in } \Omega_{+} .
$$

By taking the Dirichlet and Neumann traces of this relation, we obtain

$$
\frac{1}{2} \gamma_{D}^{+} u-D_{k_{+}} \gamma_{D}^{+} u+S_{k_{+}} \gamma_{N}^{+} u=0, \quad \frac{1}{2} \gamma_{N}^{+} u+D_{k_{+}}^{*} \gamma_{N}^{+} u-N_{k_{+}} \gamma_{D}^{+} u=0
$$

Finally, we use the boundary condition to conclude.

To prove the equivalence, it is sufficient to remark that the Dirichlet and Neumann traces of $u$ are given by

$$
\gamma_{D}^{+} u=-S_{k_{+}} q+\frac{1}{2} p+D_{k_{+}} p, \quad \gamma_{N}^{+} u=\frac{1}{2} q-D_{k_{+}}^{*} q+N_{k_{+}} p
$$

A calculation then shows that the boundary condition is fulfilled. 
In the present paper, the integral formulation (6) is written in a variational way and next is discretized thanks to the boundary element method. However, alternative approximations could also be considered as for example based on collocation techniques.

\subsection{Interior problem formulations}

The formulation used for the interior problem (3) depends on the property that the obstacle is homogeneous or heterogeneous. For a homogeneous scatterer, we can solve the interior problem (3) by using an integral equation discretized by a boundary element method (weak BEM-BEM coupling) or the finite element discretization of a volume variational formulation (weak FEM-BEM coupling). In the case of an inhomogeneous obstacle, a volume finite element discretization of the interior problem (3) is needed.

For a weak BEM-BEM coupling, the integral formulation for (3) is similar to the one for the exterior problem (4).

Proposition 4.2. A system of integral equations for solving (3) is

$$
\frac{1}{2} \rho T_{-}^{-1} \gamma_{N}^{-} u-D_{k_{-}} \gamma_{D}^{-} u+S_{k_{-}} \gamma_{N}^{-} u=\frac{1}{2} T_{-}^{-1} g, \quad \frac{1}{2 \rho} T_{-} \gamma_{D}^{-} u+D_{k_{-}}^{*} \gamma_{N}^{-} u-N_{k_{-}} \gamma_{D}^{-} u=\frac{1}{2 \rho} g .
$$

For the numerical simulations, the variational form of (7) is again used and discretized thanks to the BEM.

To derive a weak FEM-BEM coupling, the interior problem (3) is solved by a standard variational formulation.

Proposition 4.3. The weak formulation of the interior problem (3) is

$$
\forall v \in \mathrm{H}^{1}\left(\Omega_{-}\right), \quad \int_{\Omega_{-}} \rho \boldsymbol{\nabla} u \cdot \nabla \bar{v} d \Omega_{-}-\int_{\Omega_{-}} \rho k_{-}^{2} u \bar{v} d \Omega_{-}+\overline{\left\langle T_{-} \gamma_{D}^{-} u ; \gamma_{\bar{D}}^{-} v\right\rangle}=\overline{\left\langle g ; \gamma_{D}^{-} v\right\rangle} .
$$

The discretization of this variational formulation is done using second-order hierarchical finite elements [36] in order to achieve a better accuracy (by reducing the pollution error) in the high frequency regime compared to linear finite element methods [26].

\subsection{Conflicts for the choice of the optimal operators}

Let us recall that for the weak coupling (5), the optimal operators are given by

$$
T_{-}=-\Lambda_{+, k_{+}}, \quad T_{+}=-\Lambda_{-, k_{-}, \rho} .
$$

However, for the subproblems (3) and (4), the optimal operators are

$$
T_{-}=\Lambda_{-, k_{-}, \rho}, \quad T_{-}=-\rho \Lambda_{+, k_{-}}, \quad T_{+}=-\Lambda_{-, k_{+}, 1}, \quad T_{+}=\Lambda_{+, k_{+}} ;
$$

the operators for the interior problem (3) being indeed only optimal if the weak coupling (5) is of type BEM-BEM. We therefore observe a conflict appearing for the choice of the optimal operators. 
Let us recall that our first objective is to precondition the weak coupling (5). This will be illustrated later but the transmission operators 10 do not reach this objective as efficiently as for the transmission operators (9). In addition, the transmission operators (10) are interesting when one tries to iteratively solve the subproblems (3) and (4) through integral equations, for example by a Krylov solver. In this paper, we only consider the direct solution of these linear systems. Consequently, the transmission operators (9) are more adapted.

\section{Approximations of the DtN map}

Since the DtN operators are usually not available for a general shape $\Gamma$, and even if their evaluation would be computationally expensive, we propose to rather use well-suited approximations which were proven to be accurate and efficient [10] for other applications, most particularly in the high frequency regime.

\subsection{Nonlocal approximation of the DtN operators}

A well-adapted approximate exterior DtN operator derived in [2] is given by the squareroot operator

$$
\Lambda_{+, k}^{\mathrm{sq}}=i k \sqrt{I d+\operatorname{div}_{\Gamma}\left(\frac{1}{k_{\epsilon}^{2}} \nabla_{\Gamma}\right)},
$$

with $k_{\epsilon}=k+i \epsilon$. The parameter $\epsilon>0$ is optimized thanks to the expression [2] $\epsilon=0.4 k^{\frac{1}{3}} \kappa^{\frac{2}{3}}$, where $\kappa$ is the local mean curvature of $\Gamma$. Here, we simplify this choice by replacing $\kappa$ by the inverse of the radius of the smallest sphere containing $\Omega_{-}$. In (11), the operators $\nabla_{\Gamma}$ and $\operatorname{div}_{\Gamma}$ designate the surface gradient and divergence of a surface field, respectively. Let us remark that the definition (11) also includes a symmetrical Helmholtz-type operator which is well-adapted to a surface finite element approximation [2]. We will see later that the operator $\Lambda_{+, k}^{\mathrm{sq}}$ is well-suited to improve the convergence rate of the weak coupling formulation (5). Similarly, we introduce the operator $\Lambda_{+, k}^{\mathrm{sq}}$ for the interior problem

$$
\Lambda_{-, k, \rho}^{\mathrm{sq}}=-i k \rho \sqrt{I d+\operatorname{div}_{\Gamma}\left(\frac{1}{k_{\epsilon}^{2}} \nabla_{\Gamma}\right)} .
$$

Both operators are nonlocal but they nevertheless can numerically be computed almost locally thanks to Padé approximants (see subsection 5.2). If $k$ and $\rho$ are spatially variable, we will extend the above operators formally, which can be justified thanks to the theory of pseudodifferential operators and the associated microlocal analysis techniques [2].

As said above, the operators can be simulated locally thanks to Padé approximations. This is expected for the exterior operator $\Lambda_{+, k}^{\mathrm{sq}}$, most particularly for a convex domain and for the high frequency regime since the rays are locally scattered. However, for the interior problem, the transmitted waves have a nonlocal behavior since multiple scattering effects must be included. In practice, this partially limits the quality of $\Lambda_{-, k, \rho}^{\mathrm{sq}}$. For $k$ and $\rho$ constant, we propose to build a new nonlocal approximation as follows. The starting point 
is to remark that $\Lambda_{-, k, \rho}=\rho \Lambda_{-, k, 1}$ and that $\Lambda_{-, k, 1}$ and $\Lambda_{+, k}$ are linked by an integral formula. More precisely, we have the following proposition [13].

Proposition 5.1. If $k^{2}$ is not an eigenvalue of the interior Dirichlet Laplacian boundaryvalue problem, we then have

$$
S_{k}\left(\Lambda_{-, k, 1}-\Lambda_{+, k}\right)=I d .
$$

As a consequence, one gets the following relation

$$
\Lambda_{-, k, 1}=S_{k}^{-1}+\Lambda_{+, k}
$$

Concretely, this result shows that we can easily build some approximations of $\Lambda_{-, k, \rho}$ from approximations of $\Lambda_{+, k}$. It is then natural to use the square-root approximation of $\Lambda_{+, k}$ to construct the following integral operator approximation of $\Lambda_{-, k, \rho}$, denoted by $\Lambda_{-, k, \rho}^{\mathrm{int}}$,

$$
\Lambda_{-, k, \rho}^{\mathrm{int}}=\rho S_{k}^{-1}+i k \rho \sqrt{I d+\operatorname{div}_{\Gamma}\left(\frac{1}{k_{\epsilon}^{2}} \nabla_{\Gamma}\right)} .
$$

For variable $k$ and $\rho$, the relation $\Lambda_{-, k, \rho}=\rho \Lambda_{-, k, 1}$ is no longer fulfilled, limiting the above approach to $k$ and $\rho$ constant. Since the operator $\Lambda_{-, k, \rho}^{\text {int }}$ is defined through an integral operator, its evaluation requires a larger computational cost and an increased memory storage which are also a key parameter for choosing the transmission operator. Let us finally remark that $S_{k}^{-1}$ could be inverted explicitly up to a compact operator thanks to the Calderón relations, i.e. by using $-4 N_{k}$ as an approximation. Nevertheless, it appears that it strongly deteriorates the quality of the approximation when used in practice.

\subsection{Local approximation of the transmission operators}

The operator $\Lambda_{+, k}^{\mathrm{sq}}$ can first be approximated by the following zeroth-order Taylor approximation

$$
\Lambda_{+, k}^{0}=i k I d .
$$

This approximation is valid as long as the wavenumber $k$ is large enough, but should nevertheless be less accurate as $\Lambda_{+, k}^{\mathrm{sq}}$. Similarly, one gets the interior approximation of order zero

$$
\Lambda_{-, k, \rho}^{0}=-i k \rho I d
$$

To increase the accuracy of the approximate DtN map while keeping the localization property, one could alternatively use suitable rational approximations [2, 10] of the squareroot. More precisely, the $N_{p}$-th order Padé approximation of the square-root with rotating branch-cut of angle $\theta_{p}$ is given by

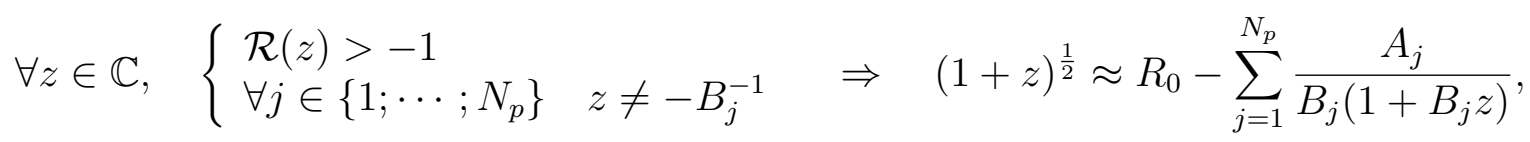


with

$$
\begin{gathered}
R_{0}=C_{0}+\sum_{j=1}^{N_{p}} \frac{A_{j}}{B_{j}}, \quad A_{j}=\frac{e^{-i \frac{\theta_{p}}{2}} a_{j}}{\left(1+b_{j}\left(e^{-i \theta_{p}}-1\right)\right)^{2}}, \quad B_{j}=\frac{e^{-i \theta_{p}} b_{j}}{1+b_{j}\left(e^{-i \theta_{p}}-1\right)}, \\
a_{j}=\frac{2}{2 N_{p}+1} \sin ^{2}\left(\frac{j \pi}{2 N_{p}+1}\right), \quad b_{j}=\cos ^{2}\left(\frac{j \pi}{2 N_{p}+1}\right), C_{0}=e^{i \frac{\theta_{p}}{2}}\left(1+\sum_{j=1}^{N_{p}} \frac{a_{j}\left(e^{-i \theta_{p}}-1\right)}{1+b_{j}\left(e^{-i \theta_{p}}-1\right)}\right) .
\end{gathered}
$$

Based on this approximation, we will consider the following formal evaluations of the squareroot operator

$$
\sqrt{I d+\operatorname{div}_{\Gamma}\left(\frac{1}{k_{\epsilon}^{2}} \nabla_{\Gamma}\right)} \approx R_{0} I d-\sum_{j=1}^{N_{p}} \frac{A_{j}}{B_{j}}\left[I d+B_{j} \operatorname{div}_{\Gamma}\left(\frac{1}{k_{\epsilon}^{2}} \nabla_{\Gamma}\right)\right]^{-1} .
$$

As a consequence, the square-root operator appearing in the approximations $\Lambda_{+, k}^{\mathrm{sq}}, \Lambda_{-, k, \rho}^{\mathrm{sq}}$ and $\Lambda_{-, k, \rho}^{\text {int }}$ of the DtN operator can be evaluated via (16), leading to new approximations of the DtN operator. They are called Padé approximations and are designated by $\Lambda_{+, k}^{\mathrm{sq}, N_{p}, \theta_{p}}, \Lambda_{-, k, \rho}^{\mathrm{sq}, N_{p}, \theta_{p}}$ and $\Lambda_{-, k, \rho}^{\mathrm{int}, N_{p}, \theta_{p}}$. These new approximations are much easier to use since their numerical evaluation can be obtained at low computational cost and memory storage. Indeed, it requires the solution to $N_{p}$ complex-valued dissipative Helmholtz-type PDEs on $\Gamma$ [10]. After discretization by a surface finite element method, $N_{p}$ indefinite complex-valued linear systems then have to be solved.

\section{Numerical results}

For all the numerical tests, BEMPP [35] has been used to build the matrices approximating the integral operators and GetDP [19] to obtain the finite element matrices. The weak iterative coupling procedure, in particular GMRES, has been implemented in Matlab. Four scatterer geometries are considered: the unit sphere, the ellipsoid of equation $\frac{x^{2}}{1.5^{2}}+\frac{y^{2}}{0.5^{2}}+\frac{z^{2}}{0.5^{2}}=1$, a cube centered at the origin and of side-length 2 , and the same cube with a reentrant corner (see Fig. 1). For all the simulations, an incident plane wave with incidence $\boldsymbol{\sigma}_{\text {inc }}=(-1,0,0)$ has been chosen. In the following, we also evaluate the far-field solution through the Sonar Cross Section (SCS) defined by

$$
\operatorname{SCS}\left(\boldsymbol{\sigma}, \boldsymbol{\sigma}_{\mathrm{inc}}\right):=10 \log _{10}\left(4 \pi \lim _{r \rightarrow+\infty} r^{2} \frac{|u(r, \boldsymbol{\sigma})|^{2}}{\left|u_{\mathrm{inc}}\right|^{2}}\right) \quad(\mathrm{dB})
$$

\subsection{Weak BEM-BEM coupling}

In the case of the weak BEM-BEM coupling, both the exterior and the interior problems, as well as the weak coupling unknowns, are discretized using $P_{1}$ triangular elements. In order to assess the numerical properties of the weak coupling, we analyze the influence of the Padé approximation, the choice of the pair of transmission operators, as well as the mesh refinement on the convergence rate of the iterative linear solver. In addition, we also evaluate the link between the tolerance of the GMRES with the accuracy of the SCS. 

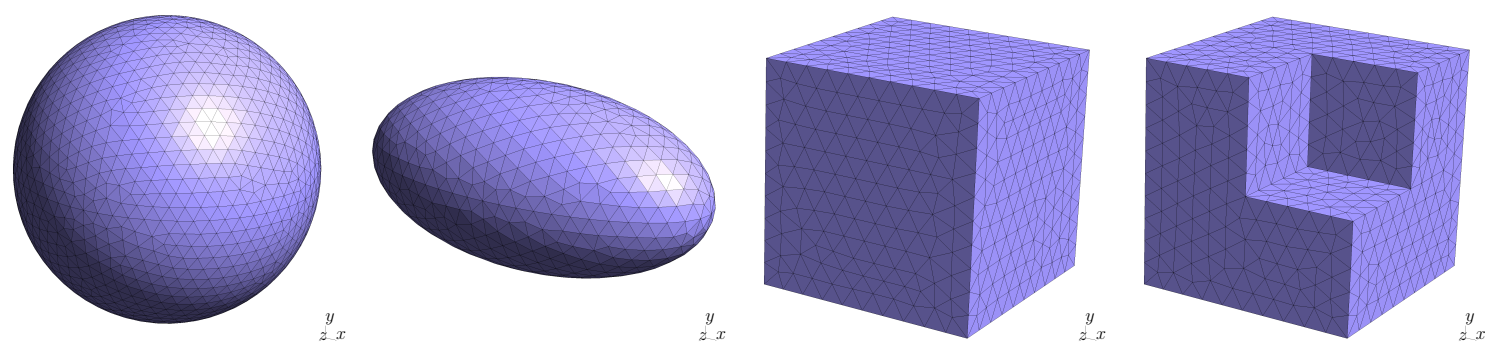

Figure 1: Scatterer geometries: sphere, ellipsoid, cube and cube with reentrant corner.

Influence of the Padé approximation. We present in Fig. 2 the evolution of the number of iterations of GMRES for the weak BEM-BEM coupling in terms of the interior wavenumber when the transmission operators correspond to the approximations $\Lambda_{+, k_{+}}^{\mathrm{sq}, N_{p}, \theta_{p}}$ and $\Lambda_{-, k_{-}, \rho}^{\mathrm{sq}, N_{p}, \theta_{p}}$ of the DtN operator. In this figure, the scatterer is the unit sphere, $k_{+}=10, \rho=5$ and several pairs of parameters $\left(N_{p} ; \theta_{p}\right)$ are considered. The GMRES tolerance is set to $10^{-6}$. In the first graph, the value of $\theta_{p}$ is fixed to $\frac{\pi}{2}$. In the second, $N_{p}$ is set to 4 . The mesh size $h$ is equal to one fifth of the smallest wavelength in the diffraction-transmission problem, i.e.:

$$
h=\frac{1}{5} \min \left(\lambda_{-} ; \lambda_{+}\right) \quad \text { with } \quad \lambda_{ \pm}=\frac{2 \pi}{k_{ \pm}} .
$$
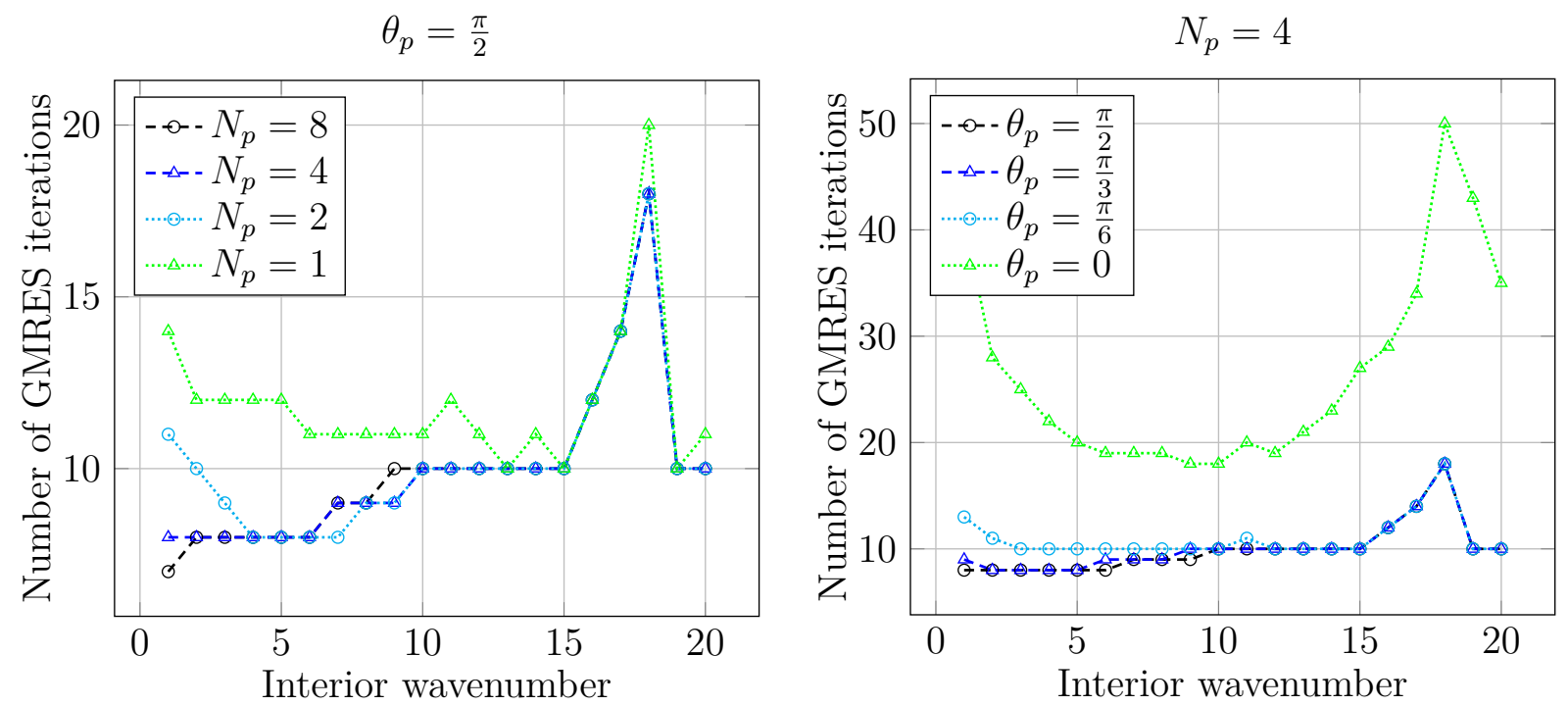

Figure 2: Influence of parameters $N_{p}$ and $\theta_{p}$ on the convergence of the weak BEM-BEM coupling.

Results from Fig. 2 indicate that it is sufficient to consider 4 terms in the Padé approximations to obtain a good GMRES convergence for the weak BEM-BEM coupling. Moreover, the optimal value for the rotation of the branch cut is $\frac{\pi}{2}$. Consequently, from now on we will always set $N_{p}=4$ and $\theta_{p}=\frac{\pi}{2}$. 
Influence of the transmission operators. Fig. 3 shows the evolution of the number of GMRES iterations vs. the interior wavenumber. For each scatterer, six curves associated each to a pair of transmission operators $\left(T_{-} ; T_{+}\right)$are plotted. The transmission operators correspond to the approximations $\Lambda_{+, k_{+}}^{\mathrm{sq}, N_{p}, \theta_{p}}, \Lambda_{+, k_{+}}^{0}, \Lambda_{-, k_{-}, \rho}^{\mathrm{int}, N_{p}, \theta_{p}}, \Lambda_{-, k_{-}, \rho}^{\mathrm{sq}, N_{p}, \theta_{p}}$ and $\Lambda_{-, k_{-}, \rho}^{0}$ of the DtN operators. The physical parameters $k_{-}, k_{+}$and $\rho$ depend on the scatterer and are indicated on the figure. The mesh size and the GMRES tolerance are set as in the previous paragraph.

Results from Fig. 3 suggest that the weak BEM-BEM coupling should be based on the following pairs of transmission operators:

$$
\left(\Lambda_{+, k_{+}}^{\mathrm{sq}, N_{p}, \theta_{p}} ; \Lambda_{-, k_{-}, \rho}^{\mathrm{sq}, N_{p}, \theta_{p}}\right), \quad\left(\Lambda_{+, k_{+}}^{\mathrm{sq}, N_{p}, \theta_{p}} ; \Lambda_{-, k_{-}, \rho}^{0}\right),
$$

in order to be robust and well-suited for large wavenumbers. Indeed, while these pairs are not optimal for all the scatterer geometries, they allow to achieve excellent GMRES convergence for all the considered objects. Moreover, while they might not be optimal for some geometries (sphere and ellipsoid), the convergence is still very good. This is linked to the fact that $\Lambda_{+, k_{+}}^{\mathrm{sq}, N_{p}, \theta_{p}}$ is an accurate approximation of $\Lambda_{+, k_{+}}$, which ensures the quasinilpotence of $S_{\pi}$. In effect, $\Lambda_{+, k_{+}}^{\mathrm{sq}, N_{p}, \theta_{p}}$ compensates the fact that $\Lambda_{-, k_{-}, \rho}^{\mathrm{sq}, N_{p}, \theta_{p}}$ and $\Lambda_{-, k_{-}, \rho}^{0}$ are quite poor approximations $\Lambda_{-, k_{-}, \rho}$.

It is interesting to notice that convergences for the pair (17) are quasi-identical when the wavenumber is large. This is due to the fact that the following approximation becomes more accurate as $k_{-}$increases:

$$
\Lambda_{-, k_{-}, \rho}^{\mathrm{sq}} \approx \Lambda_{-, k_{-}, \rho}^{0} .
$$

When the interior wavenumber is small however, $\Lambda_{-, k_{-}, \rho}^{\mathrm{sq}, N_{p}, \theta_{p}}$ leads to better results than $\Lambda_{-, k_{-}, \rho}^{0}$. In practice, one could thus envision the implementation of the weak BEM-BEM coupling using two pairs of transmission operators: one could choose $\Lambda_{-, k_{-}, \rho}^{\mathrm{sq}, N_{p}, \theta_{p}}$ as exterior transmission operator when the interior wavenumber is small to moderate; and replace $\Lambda_{-, k_{-}, \rho}^{\mathrm{sq}, N_{p}, \theta_{p}}$ by $\Lambda_{-, k_{-}, \rho}^{0}$ for large interior wavenumbers. The operator pairs (17) present another advantage that makes them particularly well suited for high-frequency calculations: they lead to the lowest dependency of the GMRES iteration count as the interior wavenumber increases.

The increase in the number of iterations depends on the scatterer geometry: it is very small for the sphere, slightly larger for the ellipsoid and significant for the cube and the cube with the reentrant corner. This is expected, as the sphere is the most favorable object for $\Lambda_{+, k_{+}}^{\mathrm{sq}}$ to be a good approximation of $\Lambda_{+, k_{+}}$: it is a smooth convex object with constant curvature. The ellipsoid is smooth and convex but with non-constant curvature, while the cube is convex but non-smooth. The cube with the reentrant corner is neither smooth nor convex. Novel high-order absorbing boundary conditions for polyhedral domains [31] could be used to improve this behavior: this is the subject of current work.

Let us now comment the results obtained with the following pairs of transmission operators:

$$
\left(\Lambda_{+, k_{+}}^{\mathrm{sq}, N_{p}, \theta_{p}} ; \Lambda_{-, k_{-}, \rho}^{\mathrm{int}, N_{p}, \theta_{p}}\right), \quad\left(\Lambda_{+, k_{+}}^{0} ; \Lambda_{-, k_{-}, \rho}^{\mathrm{int}, N_{p}, \theta_{p}}\right) .
$$

For the sphere, they lead to good convergence of the weak BEM-BEM coupling. The convergence for $\left(\Lambda_{+, k_{+}}^{0} ; \Lambda_{-, k_{-}, \rho}^{\mathrm{int}, N_{p}, \theta_{p}}\right)$ allows to confirm that $\Lambda_{-, k_{-}, \rho}^{\mathrm{int}}$ is a good approximation of $\Lambda_{-, k_{-}, \rho}$. 
Sphere

$k_{+}=10, \rho=5$

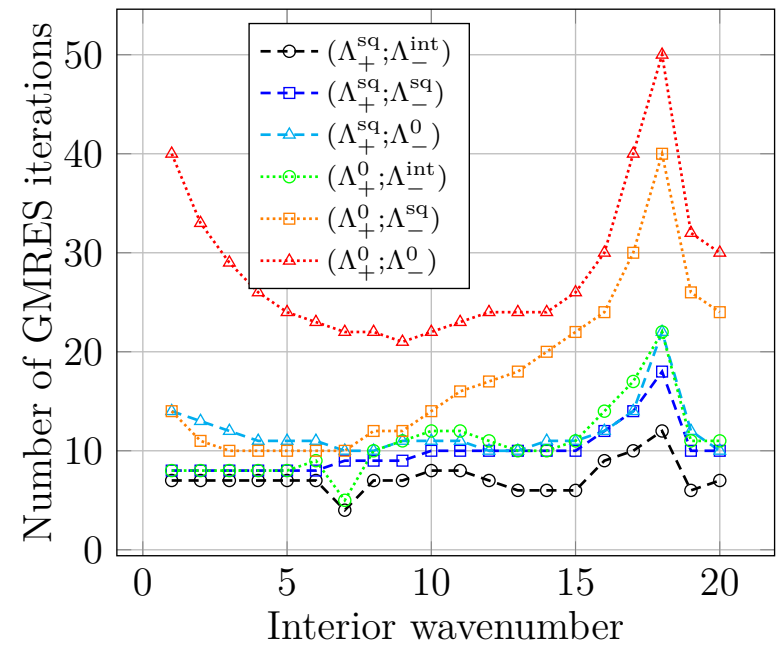

Cube

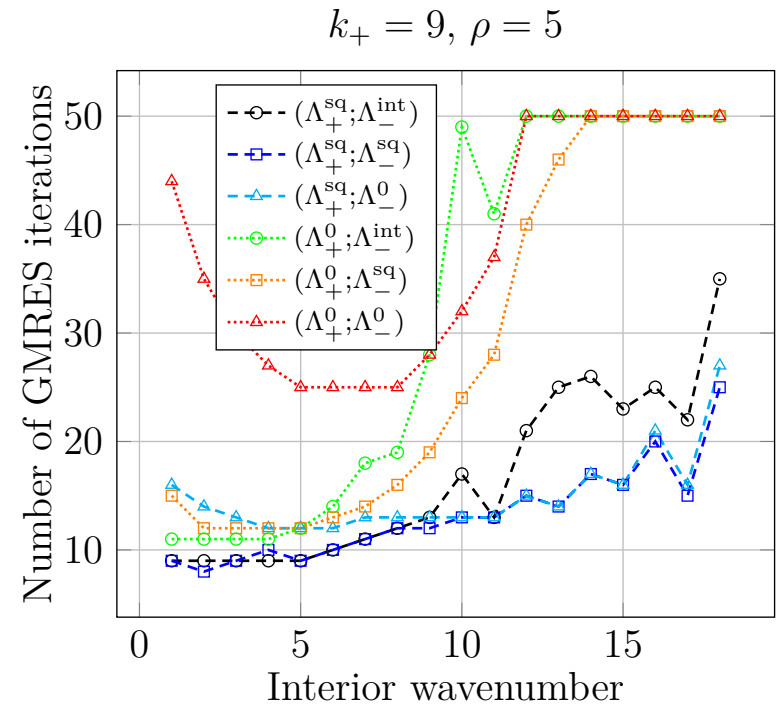

Ellipsoid

$k_{+}=10, \rho=5$

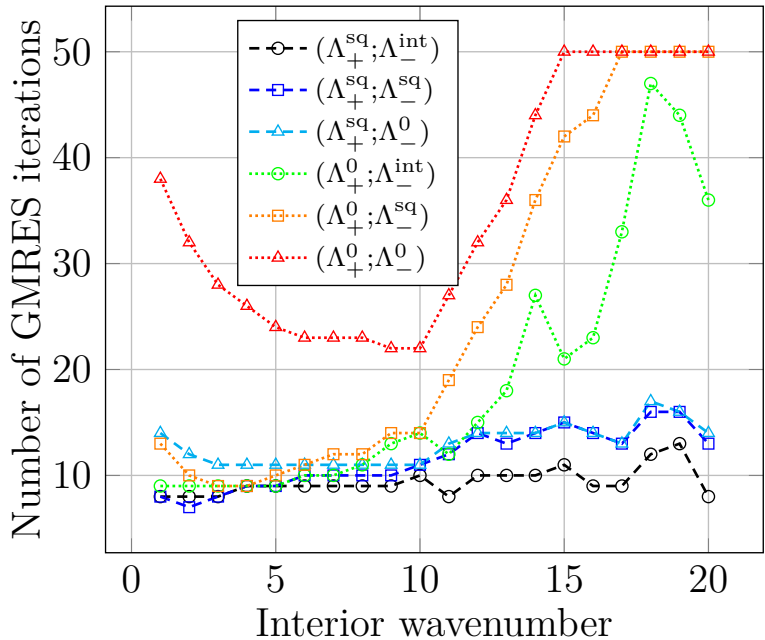

Cube with rentrent corner

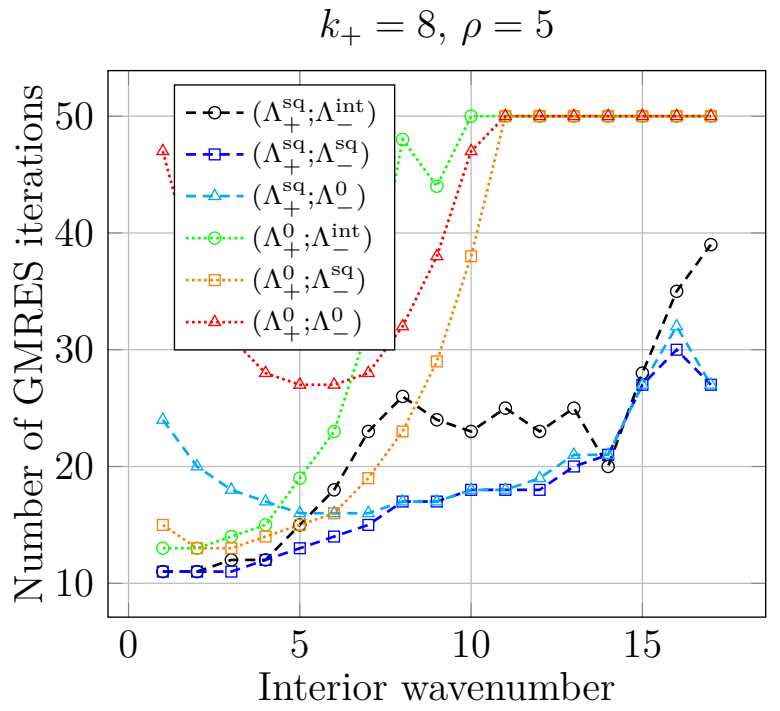

Figure 3: Number of GMRES iterations for the weak BEM-BEM coupling vs. the interior wavenumber. 
Indeed, $\Lambda_{+, k_{+}}^{0}$ is not a sufficiently good approximation of $\Lambda_{+, k_{+}}$to make $S_{\pi}$ quasi-nilpotent. For the ellipsoid, the cube and the cube with reentrant corner, the results obtained with the pair (18) are not as convincing. (Note that the maximum number of GMRES iterations was set to 50, which explains the plateaus in the curves.)

In order to explain the results obtained for the ellipsoid, the cube and the cube with the reentrant corner, recall that the approximation $\Lambda_{-, k_{-}, \rho}^{\mathrm{int}}$ is constructed as follows:

$$
\Lambda_{-, k_{-}, \rho}^{\mathrm{int}}=\rho\left(S_{k_{-}}^{-1}+\Lambda_{+, k_{-}}^{\mathrm{sq}}\right) .
$$

Moreover, we know that the quality of $\Lambda_{-, k_{-}, \rho}^{\mathrm{int}}$ as an approximation of $\Lambda_{-, k_{-}, \rho}$ is directly linked to the quality of $\Lambda_{+, k_{-}}^{\mathrm{sq}}$ as an approximation of $\Lambda_{+, k_{-}}$. For the cube and the cube with reentrant corner, we have established that $\Lambda_{+, k_{-}}^{\mathrm{sq}}$ is not a good approximation of $\Lambda_{+, k_{-}}$. It is thus normal that $\Lambda_{-, k_{-}, \rho}^{\mathrm{int}, N_{p}, \theta_{p}}$ does not approximate $\Lambda_{-, k_{-}, \rho}$ very accurately. It is however surprising that the pair $\left(\Lambda_{+, k_{+}}^{\mathrm{sq}, N_{p}, \theta_{p}} ; \Lambda_{-, k_{-}, \rho}^{\mathrm{int}, N_{p}, \theta_{p}}\right)$ leads to a worse convergence than $\left(\Lambda_{+, k_{+}}^{\mathrm{sq}, N_{p}, \theta_{p}} ; \Lambda_{-, k_{-}, \rho}^{\mathrm{sq}, N_{p}, \theta_{p}}\right)$. This suggests that the quality of the approximations of $\Lambda_{-, k_{-}, \rho}$ obtained from

$$
\Lambda_{-, k_{-}, \rho}=\rho\left(S_{k_{-}}^{-1}+\Lambda_{+, k_{-}}\right),
$$

strongly depends on the quality of the approximation used for $\Lambda_{+, k_{-}}$. More precisely, a small error in the approximation of $\Lambda_{+, k_{-}}$leads to a much larger error in the approximation of $\Lambda_{-, k_{-}, \rho}$. Finally, for the ellipsoid we have already mentioned that $\Lambda_{+, k_{-}}^{\mathrm{sq}}$ is a suitable approximation of $\Lambda_{+, k_{-}}$, albeit slightly worse than for the sphere. The results obtained for the pair $\left(\Lambda_{+, k_{+}}^{0} ; \Lambda_{-, k_{-}, \rho}^{\mathrm{int}, N_{p}, \theta_{p}}\right)$ seem to indicate that this is sufficient to degrade $\Lambda_{-, k_{-}, \rho}^{\mathrm{int}}$. However, this degradation is quite moderate since the pair $\left(\Lambda_{+, k_{+}}^{\mathrm{sq}, N_{p}, \theta_{p}} ; \Lambda_{-, k_{-}, \rho}^{\mathrm{int}, N_{p}, \theta_{p}}\right)$ gives the best GMRES convergence. Concretely, $\Lambda_{+, k_{+}}^{\mathrm{sq}}$ allows to compensate the degradation of $\Lambda_{-, k_{-}, \rho}^{\mathrm{int}}$.

Finally, the transmission condition pairs

$$
\left(\Lambda_{+, k_{+}}^{0} ; \Lambda_{-, k_{-}, \rho}^{0}\right), \quad\left(\Lambda_{+, k_{+}}^{0} ; \Lambda_{-, k_{-}, \rho}^{\mathrm{sq}, N_{p}, \theta_{p}}\right),
$$

are not well-suited for the weak BEM-BEM coupling in the high-frequency case. Indeed, the number of GMRES iterations strongly increases with this pair for all geometries. This is due to the fact that $\Lambda_{+, k_{+}}^{0}, \Lambda_{-, k_{-}, \rho}^{0}$ and $\Lambda_{-, k_{-}, \rho}^{\mathrm{sq}}$ are not accurate approximations of $\Lambda_{+, k_{+}}$ and $\Lambda_{-, k_{-}, \rho}$.

Fig. 4 shows the GMRES convergence history for the weak BEM-BEM coupling. Convergence histories are useful to estimate the number of GMRES iterations necessary to reach a prescribed relative residual. For example, for the following transmission operator pairs:

$$
\left(\Lambda_{+, k_{+}}^{\mathrm{sq}, N_{p}, \theta_{p}} ; \Lambda_{-, k_{-}, \rho}^{\mathrm{sq}, N_{p}, \theta_{p}}\right), \quad\left(\Lambda_{+, k_{+}}^{\mathrm{sq}, N_{p}, \theta_{p}} ; \Lambda_{-, k_{-}, \rho}^{0}\right),
$$

the convergence rate seems to be identical for each iteration, while for

$$
\left(\Lambda_{+, k_{+}}^{\mathrm{sq}, N_{p}, \theta_{p}} ; \Lambda_{-, k_{-}, \rho}^{\mathrm{int}, N_{p}, \theta_{p}}\right), \quad\left(\Lambda_{+, k_{+}}^{0} ; \Lambda_{-, k_{-}, \rho}^{\mathrm{int}, N_{p}, \theta_{p}}\right),
$$

the rate strongly decreases after the first iteration, most dramatically for the ellipsoid, the cube and the cube with reentrant corner. 
Sphere

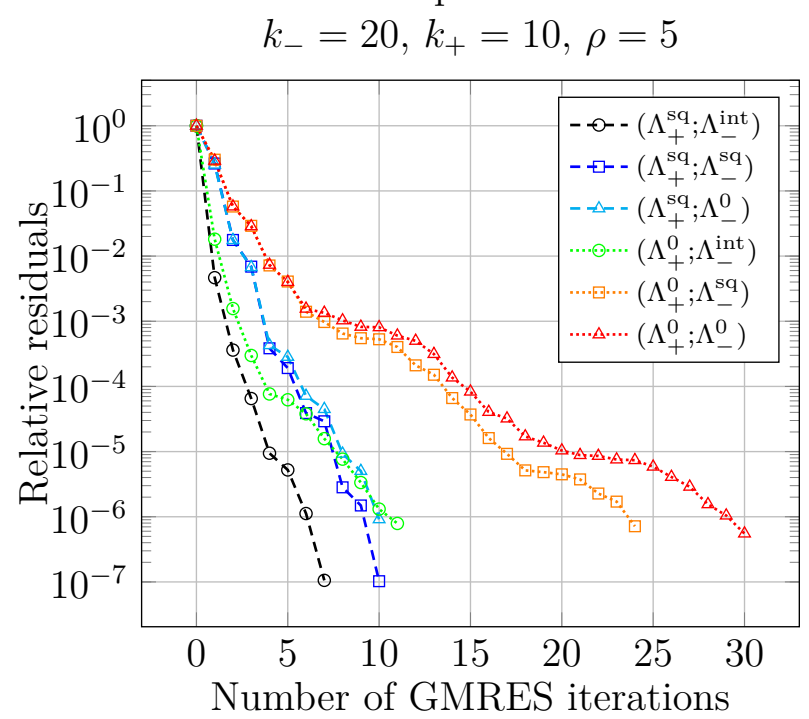

Cube

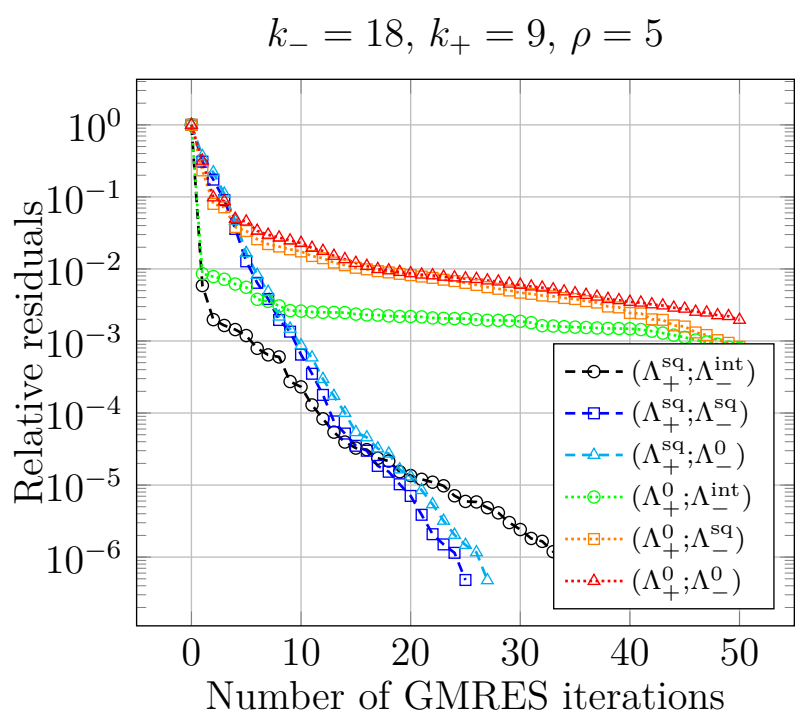

Ellipsoid

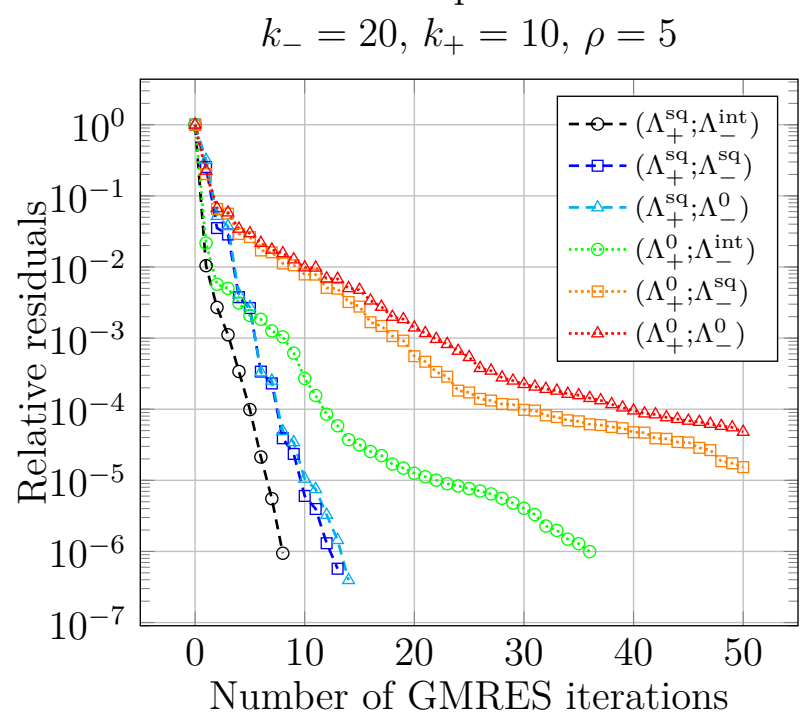

Cube with a reentrant corner $k_{-}=17, k_{+}=8, \rho=5$

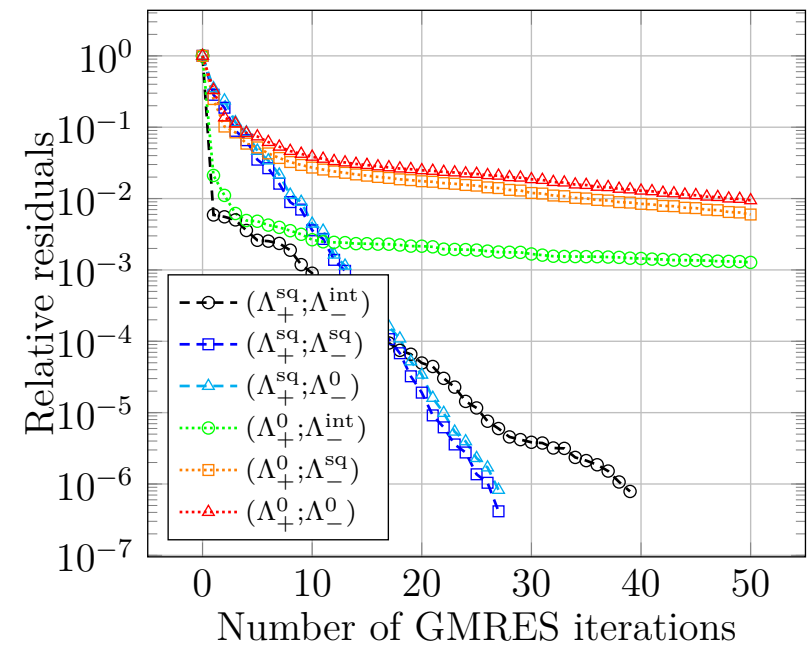

Figure 4: GMRES convergence histories for the weak BEM-BEM coupling. 
Influence of mesh refinement. Fig. 5 shows the evolution of the number of GMRES iterations in terms of mesh refinement, quantified in terms of the number of points per wavelength $n_{\lambda}$. More precisely, the mesh size is given by:

$$
h=\frac{1}{n_{\lambda}} \min \left(\lambda_{-} ; \lambda_{+}\right) \quad \text { with } \quad \lambda_{ \pm}=\frac{2 \pi}{k_{ \pm}} .
$$

For the following pairs of transmission operators

$$
\left(\Lambda_{+, k_{+}}^{\mathrm{sq}, N_{p}, \theta_{p}} ; \Lambda_{-, k_{-}, \rho}^{\mathrm{sq}, N_{p}, \theta_{p}}\right), \quad\left(\Lambda_{+, k_{+}}^{\mathrm{sq}, N_{p}, \theta_{p}} ; \Lambda_{-, k_{-}, \rho}^{\mathrm{int}, N_{p}, \theta_{p}}\right), \quad\left(\Lambda_{+, k_{+}}^{0} ; \Lambda_{-, k_{-}, \rho}^{\mathrm{sq}, N_{p}, \theta_{p}}\right), \quad\left(\Lambda_{+, k_{+}}^{0} ; \Lambda_{-, k_{-}, \rho}^{\mathrm{int}, N_{p}, \theta_{p}}\right),
$$

mesh refinement does not significantly degrade the GMRES convergence, while convergence clearly deteriorates with the pairs:

$$
\left(\Lambda_{+, k_{+}}^{\mathrm{sq}, N_{p}, \theta_{p}} ; \Lambda_{-, k_{-}, \rho}^{0}\right), \quad\left(\Lambda_{+, k_{+}}^{0} ; \Lambda_{-, k_{-}, \rho}^{0}\right) .
$$

As a consequence, to construct a robust weak BEM-BEM coupling, it seems preferable to avoid "mixing" transmission operators

$$
\left(\Lambda_{+, k_{+}}^{\mathrm{sq}, N_{p}, \theta_{p}} ; \Lambda_{-, k_{-}, \rho}^{\mathrm{sq}, N_{p}, \theta_{p}}\right), \quad\left(\Lambda_{+, k_{+}}^{\mathrm{sq}, N_{p}, \theta_{p}} ; \Lambda_{-, k_{-}, \rho}^{0}\right),
$$

and limit the choice to the following pair of transmission operators:

$$
\left(\Lambda_{+, k_{+}}^{\mathrm{sq}, N_{p}, \theta_{p}} ; \Lambda_{-, k_{-}, \rho}^{\mathrm{sq}, N_{p}, \theta_{p}}\right) .
$$

Influence of the GMRES tolerance. Figures 6 and 7 show the bistatic SCS for the sphere, the ellipsoid, the cube and the cube with reentrant corner obtained with the recommended pair of transmission operators:

$$
\left(\Lambda_{+, k_{+}}^{\mathrm{sq}, N_{p}, \theta_{p}} ; \Lambda_{-, k_{-}, \rho}^{\mathrm{sq}, N_{p}, \theta_{p}}\right) .
$$

For each considered geometry, two graphs are provided. The first represents the evolution of the bistatic SCS in the $z=0$ plane, the polar angle varying from $0^{\circ}$ to $360^{\circ}$ with a GMRES tolerance equal to $10^{-4}$. The second graph presents bistatic SCS curves on small angular ranges, obtained with different GMRES tolerances. As expected, the results confirm that the SCS converges when the GMRES tolerance decreases. More importantly, the results show that the GMRES tolerance should not exceed $10^{-3}$ to correctly estimate the SCS. In view of the GMRES convergence histories presented in Fig. 4, this allows us to conclude that sufficient accuracy on the SCS can be achieved for all the considered geometries with between 5 and 10 GMRES iterations - a very promising results for the engineering usefulness of the proposed weak coupling strategy.

\subsection{Weak FEM-BEM coupling}

For the weak FEM-BEM coupling, we use again P1 triangular finite elements for BEM and the weak coupling unknowns, and hierarchical second-order elements for FEM. The coupling between the second-order and first-order unknowns is done naturally thanks to the 
Sphere

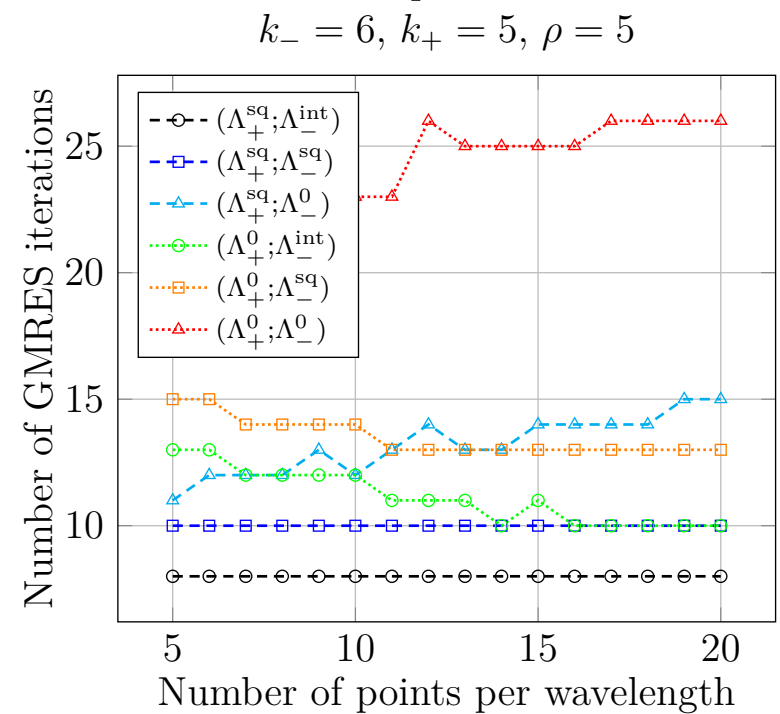

Cube

$k_{-}=6, k_{+}=5, \rho=5$

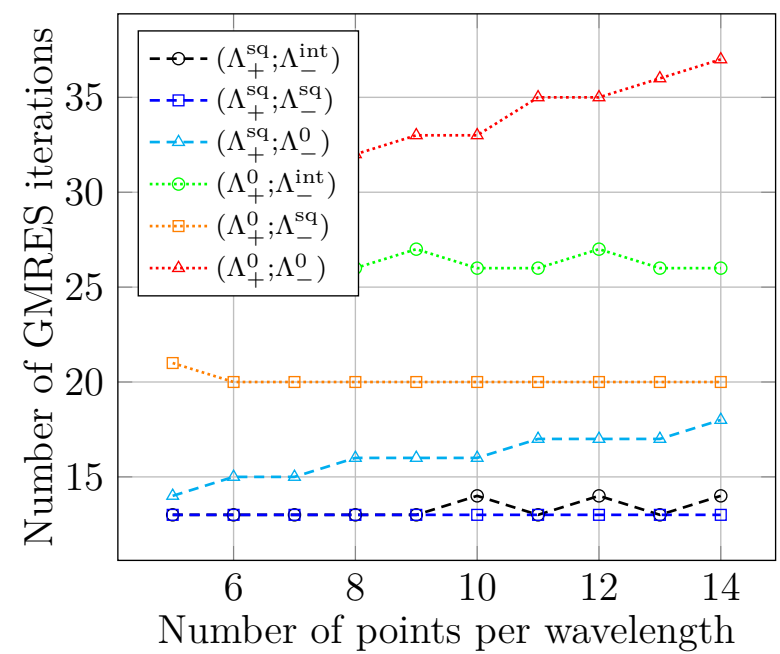

Ellipsoid

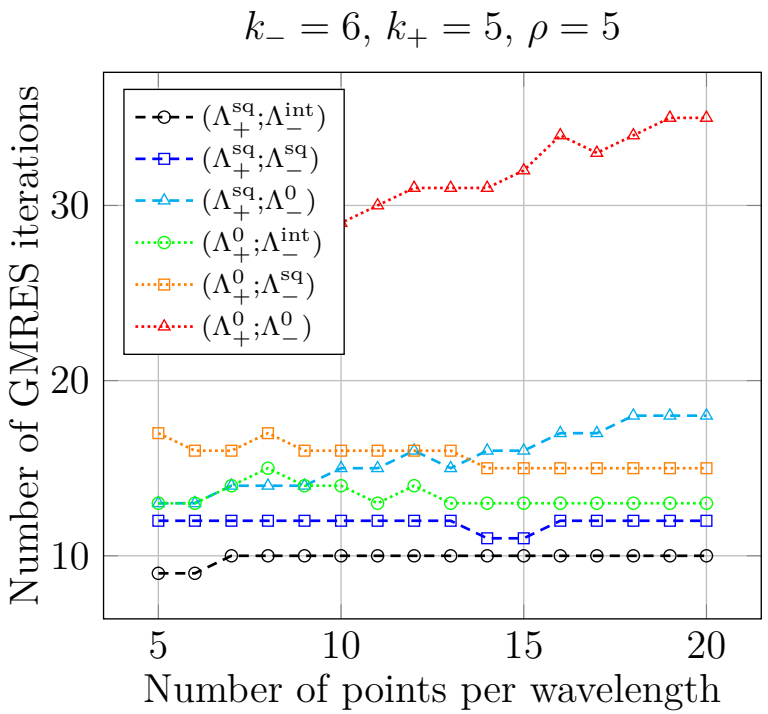

Cube with rentrent corner $k_{-}=6, k_{+}=5, \rho=5$

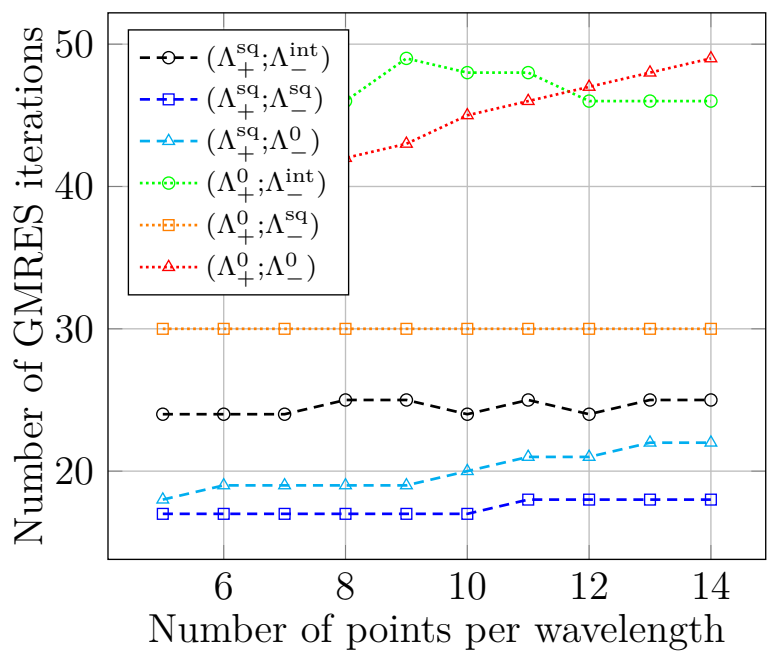

Figure 5: Number of GMRES iterations for the weak BEM-BEM coupling vs. mesh refinement. 
Sphere with tolerance of $10^{-4}$

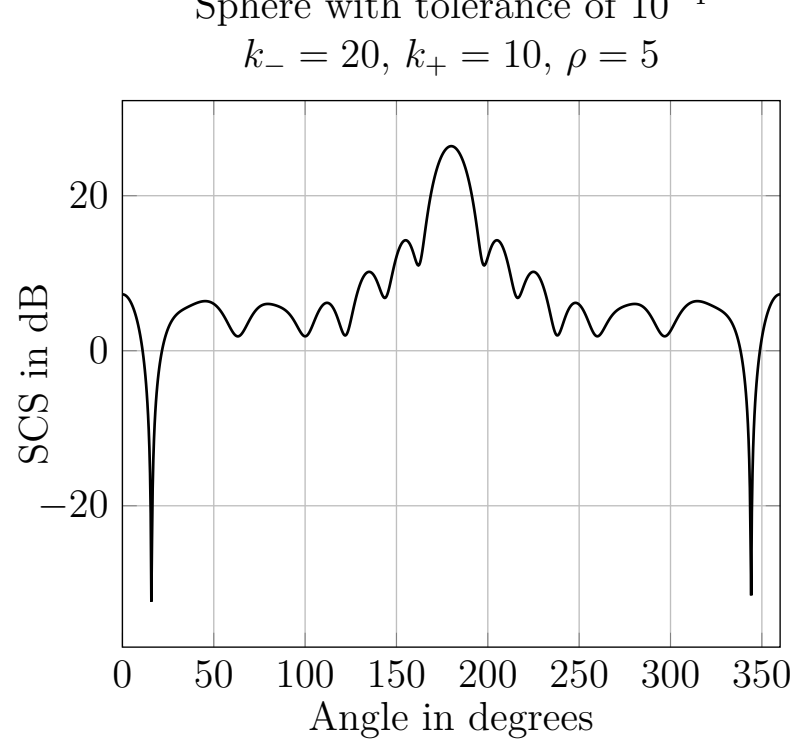

Ellipsoid with tolerance of $10^{-4}$

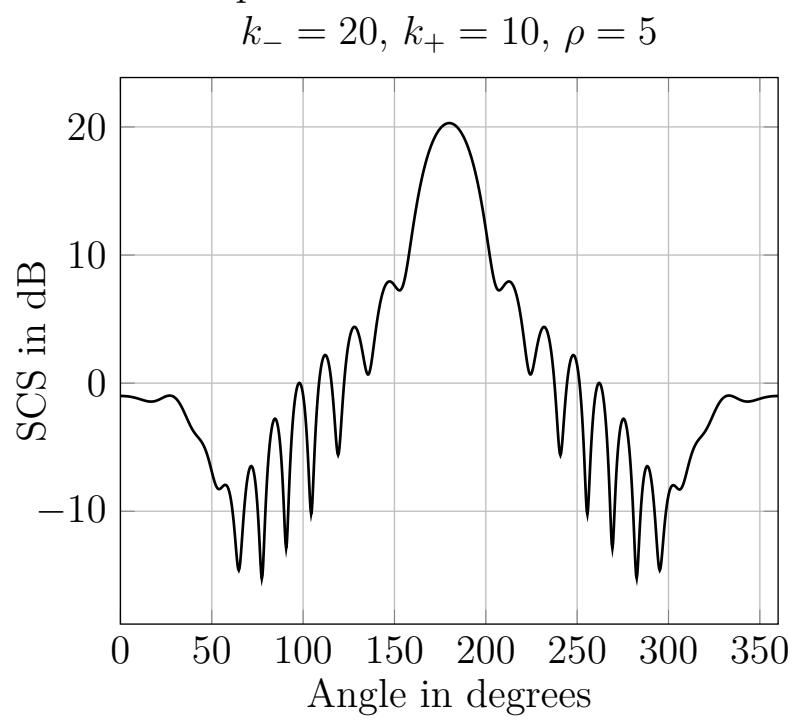

Sphere with several tolerances

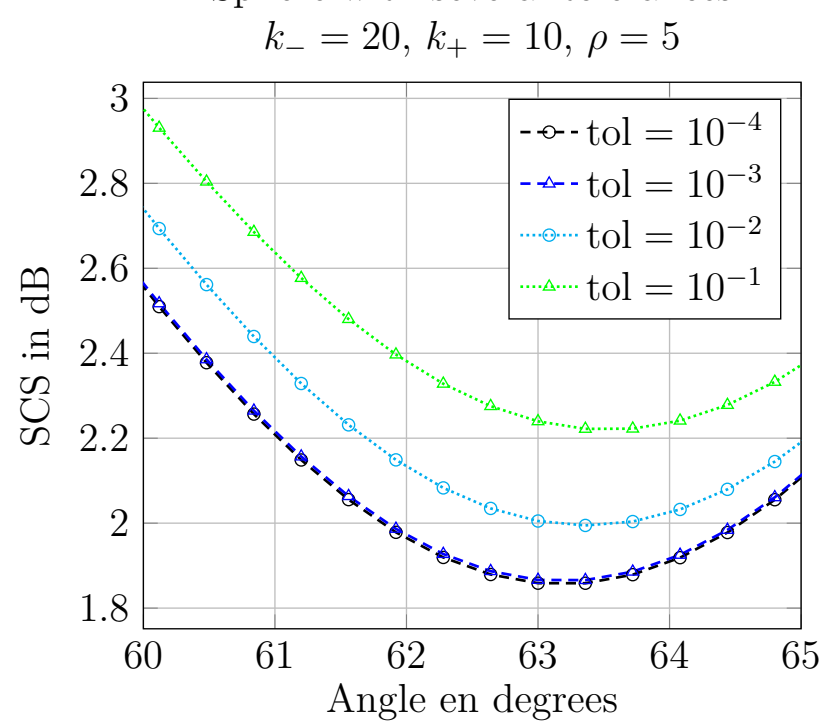

Ellipsoid with several tolerances

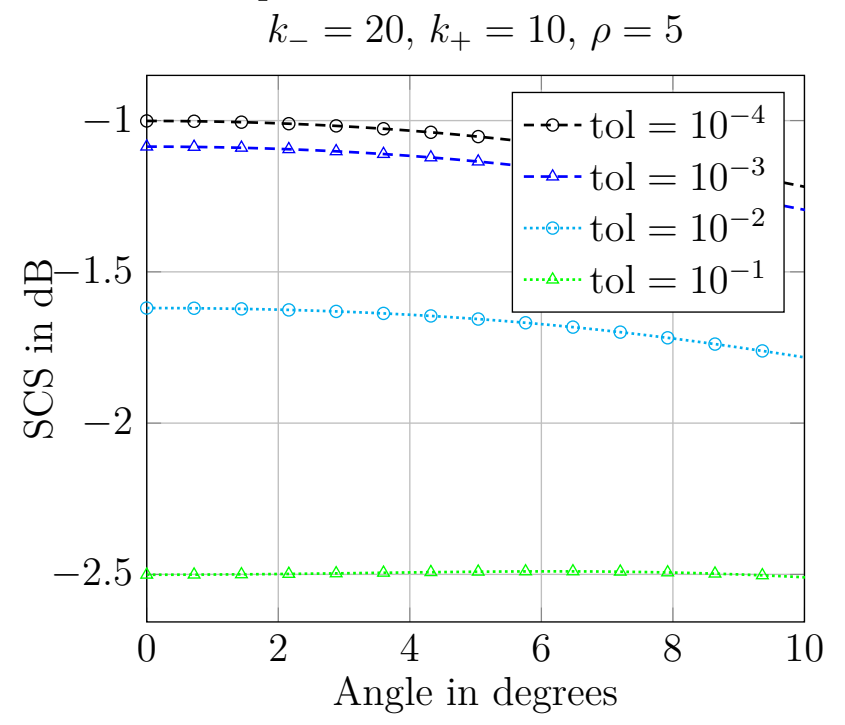

Figure 6: Convergence of the SCS with respect to the GMRES tolerance for the weak BEM-BEM coupling (sphere and ellipsoid) 
Cube with tolerance of $10^{-4}$

$k_{-}=18, k_{+}=9, \rho=5$

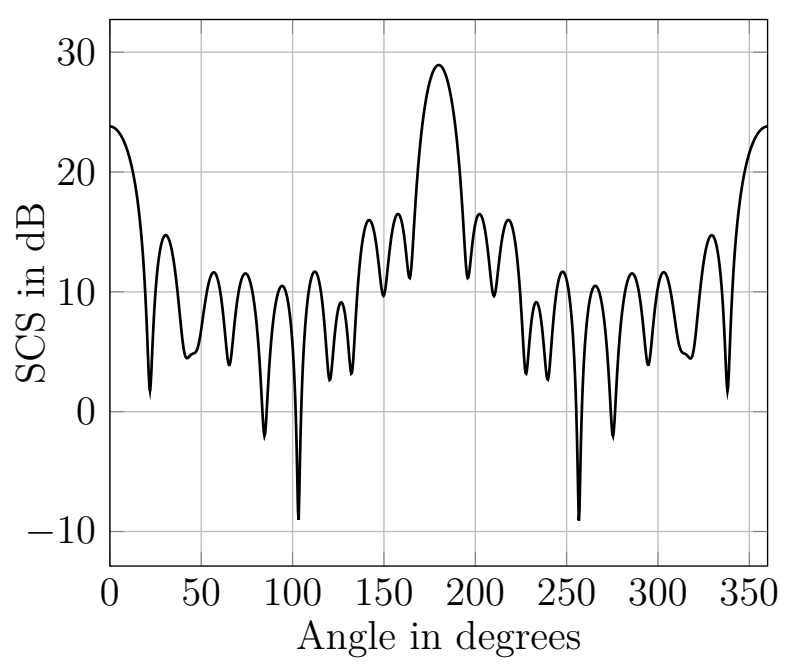

Cube with rentrent corner

with tolerance of $10^{-4}$

$k_{-}=17, k_{+}=8, \rho=5$

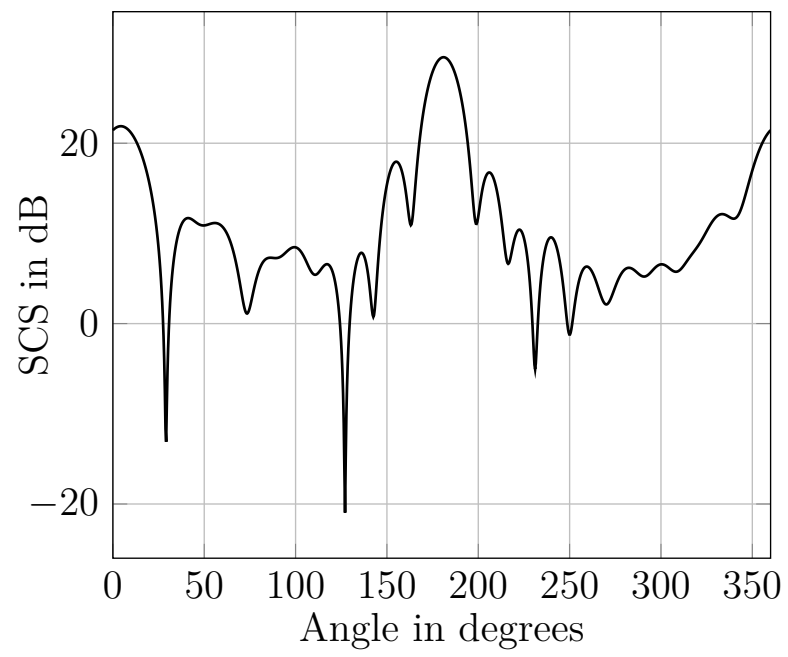

Cube with several tolerances

$k_{-}=18, k_{+}=9, \rho=5$

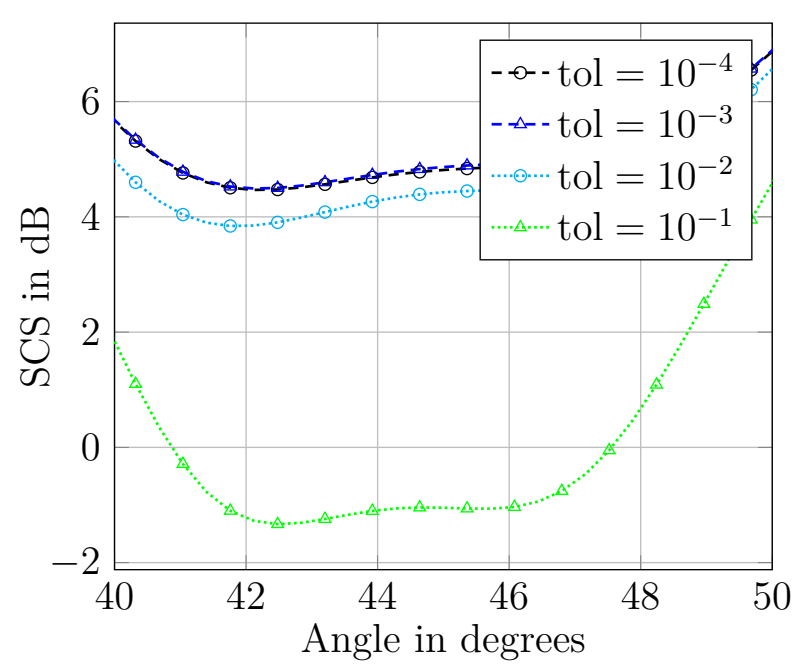

Cube with rentrent corner with several tolerances $k_{-}=17, k_{+}=8, \rho=5$

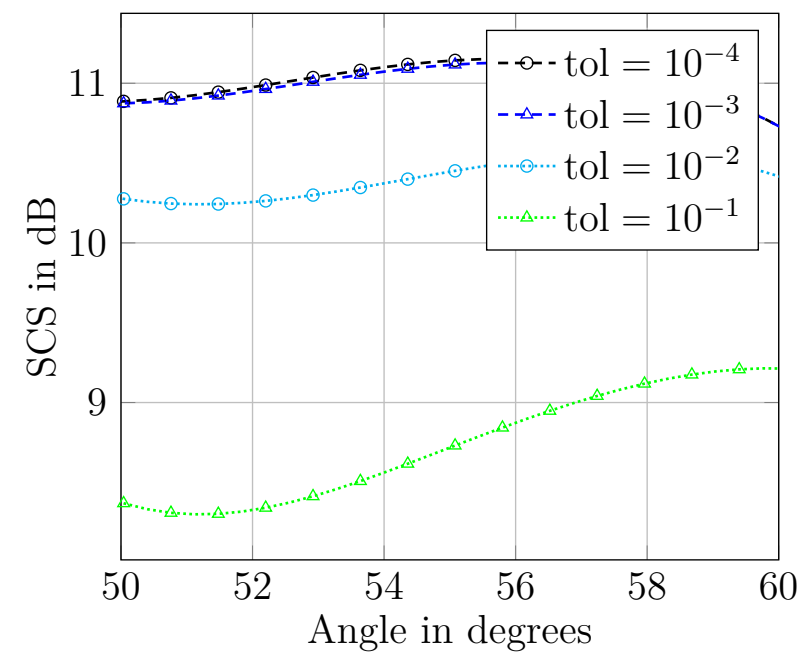

Figure 7: Convergence of the SCS with respect to the GMRES tolerance for the weak BEM-BEM coupling (cube and cube with reentrant corner) 
hierarchical basis, by setting to zero the edge degrees of freedom of the FEM unknowns on the coupling interface $\Gamma$. We restrict our study to the inhomogeneous case to validate the optimized FEM-BEM formulation. The four scatterers are the same as for the BEM-BEM coupling. However, we consider $k_{-}$and $\rho$ as the functions given by

$$
k_{-}(\mathbf{x})=k_{-, 0} e^{-\|\mathbf{x}\|^{2}}, \quad \rho(\mathbf{x})=2 \rho_{0} e^{-\ln 2 \frac{\|\mathbf{x}\|^{2}}{r_{\max }^{2}}},
$$

where $r_{\max }$ is the radius of the scatterer, i.e. $r_{\max }=\max _{\mathbf{x} \in \Omega_{-}}\|\mathbf{x}\|$. Concerning the sphere, the ellipsoid and the cubes, $r_{\max }$ is respectively equal to $1,1.5$ and $\sqrt{3}$. In addition, $\rho$ is equal to $2 \rho_{0}$ at the origin and to $\rho_{0}$ for the points of $\Omega_{-}$which are further from the origin, and $k_{-, 0}$ and $2 \rho_{0}$ correspond to the maximal values of the functions $k_{-}$and $\rho$, respectively.

Influence of the transmission operators. In Figure 8, we report the number of GMRES iterations required for solving the weak FEM-BEM coupling vs. $k_{-, 0}$. The GMRES tolerance is fixed to $10^{-6}$ and the meshsize is one fifth of the smallest wavelength, i.e.

$$
h=\frac{1}{5} \min \left(\lambda_{-} ; \lambda_{+}\right) \quad \text { with } \quad \lambda_{-}=\frac{2 \pi}{\max \left(k_{-}\right)}=\frac{2 \pi}{k_{-, 0}} \quad, \quad \lambda_{+}=\frac{2 \pi}{k_{+}} .
$$

The results are in accordance with the ones for the homogeneous case solved through the optimized weak BEM-BEM coupling. The best convergence rate is obtained for the pair of transmission operators

$$
\left(\Lambda_{+, k_{+}}^{\mathrm{sq}, N_{p}, \theta_{p}} ; \Lambda_{-, k_{-}, \rho}^{\mathrm{sq}, N_{p}, \theta_{p}}\right) .
$$

Let us remark that, when the interior wavenumber is larger, the curves associated with the pairs of transmission operators

$$
\left(\Lambda_{+, k_{+}}^{\mathrm{sq}, N_{p}, \theta_{p}} ; \Lambda_{-, k_{-}, \rho}^{\mathrm{sq}, N_{p}, \theta_{p}}\right), \quad\left(\Lambda_{+, k_{+}}^{\mathrm{sq}, N_{p}, \theta_{p}} ; \Lambda_{-, k_{-}, \rho}^{0}\right),
$$

are much more distant from each other than for the weak BEM-BEM coupling. Indeed, for example for the sphere, the value of $k_{-}$on $\Gamma$ is given by $k_{-, 0} e^{-1} \approx 0.37 k_{-, 0}$, which is too small to have an accurate approximation

$$
\Lambda_{-, k_{-}, \rho}^{\mathrm{sq}, N_{p}, \theta_{p}} \approx \Lambda_{-, k_{-}, \rho}^{0}
$$

In the previous section, we have observed that, for some pairs of transmission operators, the number of GMRES iterations for solving the weak BEM-BEM coupling significantly increases with respect to the interior wave number. This is weaker here on Figure 8 . However, this is probably more related to our specific test case than to a general situation. As an informal remark, it seems that the convergence rate of the GMRES is much more sensitive to the values of $k_{-}$on $\Gamma$ than globally in $\Omega_{-}$.

Influence of mesh refinement. Figure 9 presents the effect of the mesh refinement over the number of GMRES iterations (with tolerance $10^{-6}$ ) for the weak FEM-BEM coupling. The meshsize is defined by the number of points per wavelength following

$$
h=\frac{1}{n_{\lambda}} \min \left(\lambda_{-} ; \lambda_{+}\right) \quad \text { with } \quad \lambda_{-}=\frac{2 \pi}{\max \left(k_{-}\right)}=\frac{2 \pi}{k_{-, 0}} \quad, \quad \lambda_{+}=\frac{2 \pi}{k_{+}} .
$$


Sphere

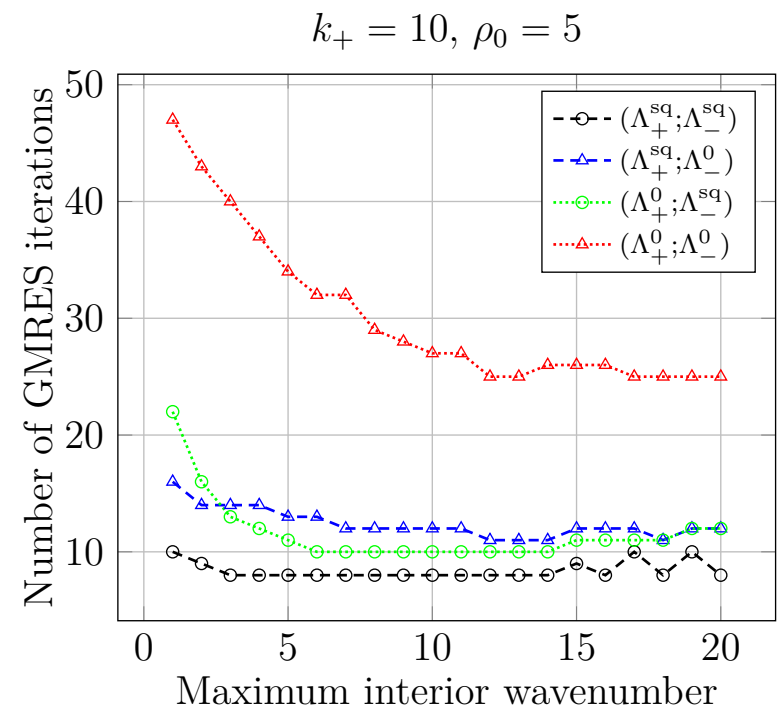

Cube

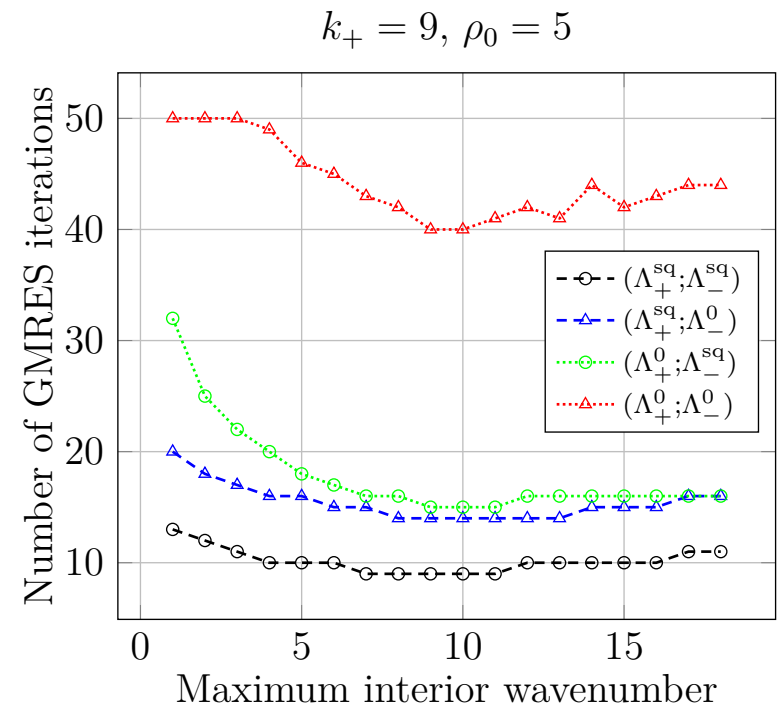

Ellipsoid

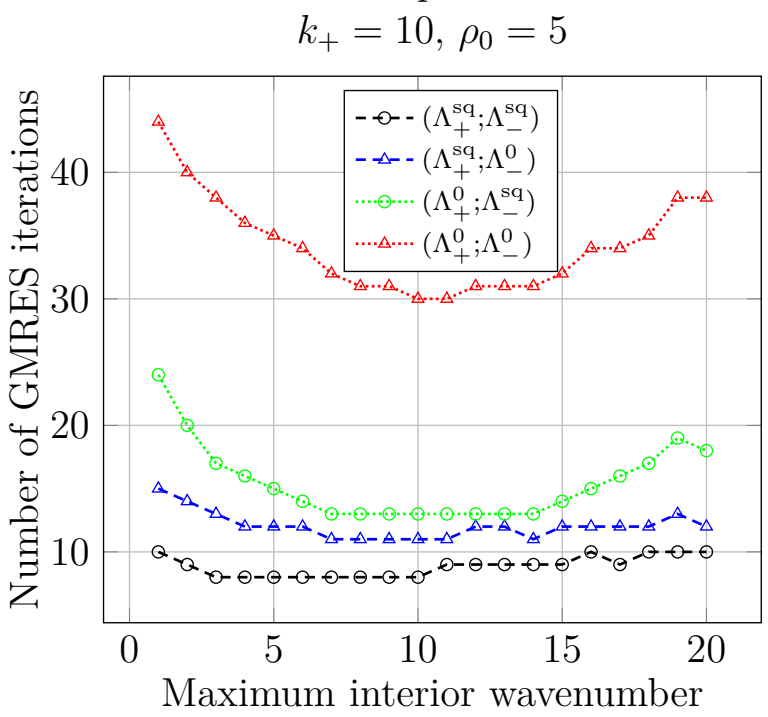

Cube with reentrant corner $k_{+}=8, \rho_{0}=5$

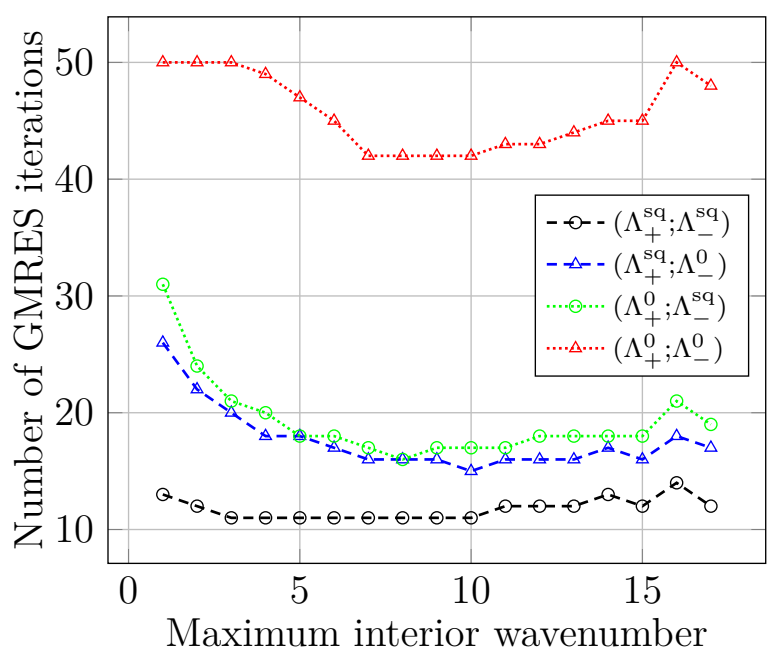

Figure 8: Number of GMRES iterations for the weak FEM-BEM coupling vs. the interior wavenumber. 
Conversely to the case of the weak BEM-BEM coupling, the number of iterations varies more with the mesh refinement when using the pair of transmission operators

$$
\left(\Lambda_{+, k_{+}}^{\mathrm{sq}, N_{p}, \theta_{p}} ; \Lambda_{-, k_{-}, \rho}^{\mathrm{sq}, N_{p}, \theta_{p}}\right) .
$$

This sensitivity is probably due to the fact that the quality of the approximation of $\Lambda_{-, k_{-}, \rho}$ by $\Lambda_{-, k_{-}, \rho}^{\mathrm{sq}, N_{p}, \theta_{p}}$ deteriorates for variable $k_{-}$and $\rho$. Nevertheless, this should not be too much penalizing in a practical computation.

Influence of the GMRES tolerance. To end, Figures 10 and 11 report the SCS obtained by the weak FEM-BEM coupling and illustrate the accuracy vs. the GMRES tolerance. They are obtained by fixing $n_{\lambda}=5$ for the pair of transmission operators

$$
\left(\Lambda_{+, k_{+}}^{\mathrm{sq}, N_{p}, \theta_{p}} ; \Lambda_{-, k_{-}, \rho}^{\mathrm{sq}, N_{p}, \theta_{p}}\right) .
$$

These results show that the tolerance of the FEM-BEM coupling, similarly to the BEM-BEM case, must be smaller than $10^{-3}$ for an accurate SCS computation.

\section{Conclusion}

In this paper, we have presented an optimized weak coupling of boundary element and finite element methods to solve acoustic scattering problems. The weak coupling was formulated as a non-overlapping Schwarz domain decomposition method, where the transmission conditions are constructed through Padé localized approximations of the Dirichletto-Neumann map. Several choices of transmission conditions were analyzed on several three-dimensional examples, highlighting the potential of the proposed approach for highfrequency scattering simulations. The extension to electromagnetic scattering is currently being developed.

\section{Acknowledgements}

The authors thank the support of Thales through the CIFRE contract 2015/0197. This work was funded in part through an ARC grant for Concerted Research Actions (ARC WAVES 15/19-03), financed by the Wallonia-Brussels Federation of Belgium.

\section{References}

[1] X. Antoine, H. Barucq, and A. Bendali. Bayliss-Turkel-like radiation conditions on surfaces of arbitrary shape. Journal of Mathematical Analysis and Applications, 229(1):184-211, 1999.

[2] X. Antoine, M. Darbas, and Y. Y. Lu. An improved surface radiation condition for high-frequency acoustic scattering problems. Computer Methods in Applied Mechanics and Engineering, 195(33-36):40604074, 2006.

[3] A. Bachelot and V. Lange. Time dependent integral method for Maxwell's system with impedance boundary condition. WIT Transactions on Modelling and Simulation, 11, 1995.

[4] A. Bayliss, M. Gunzburger, and E. Turkel. Boundary conditions for the numerical solution of elliptic equations in exterior regions. SIAM Journal on Applied Mathematics, 42(2):430-451, 1982. 
Sphere

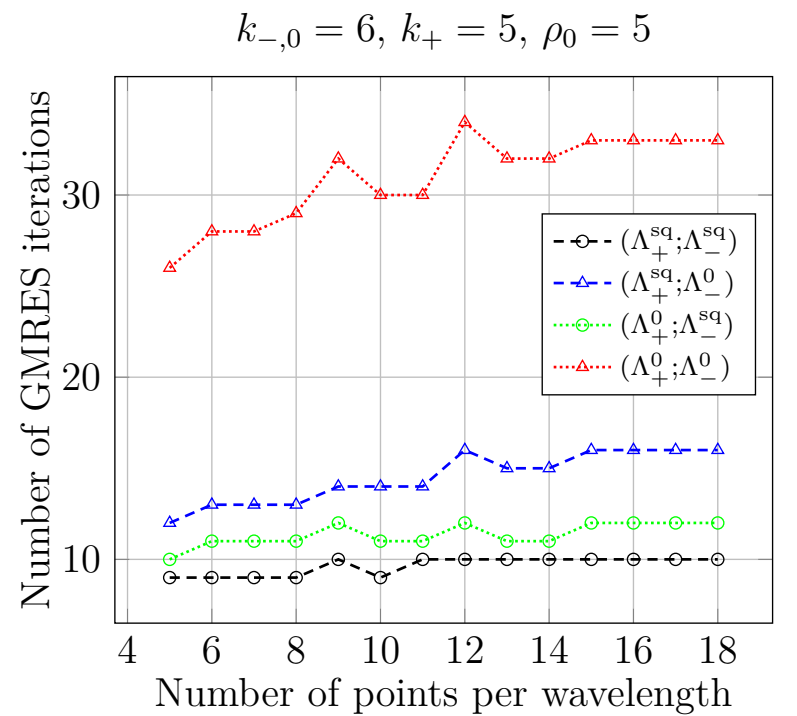

Cube

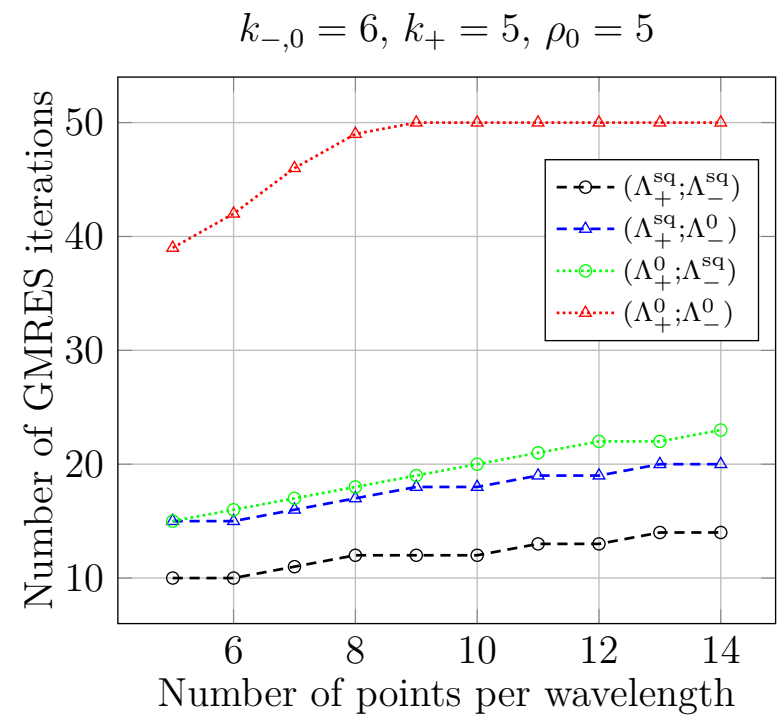

Ellipsoid

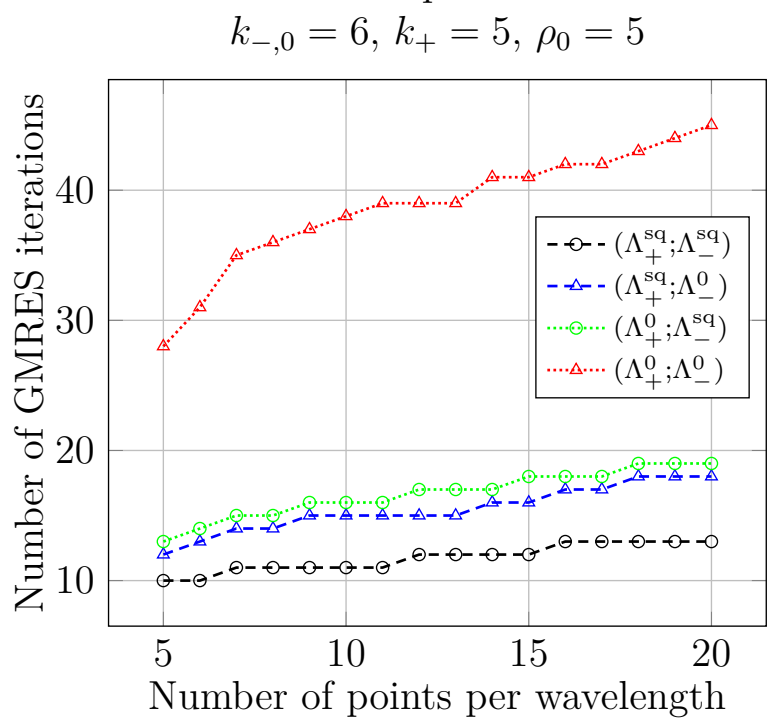

Cube with reentrant corner $k_{-, 0}=6, k_{+}=5, \rho_{0}=5$

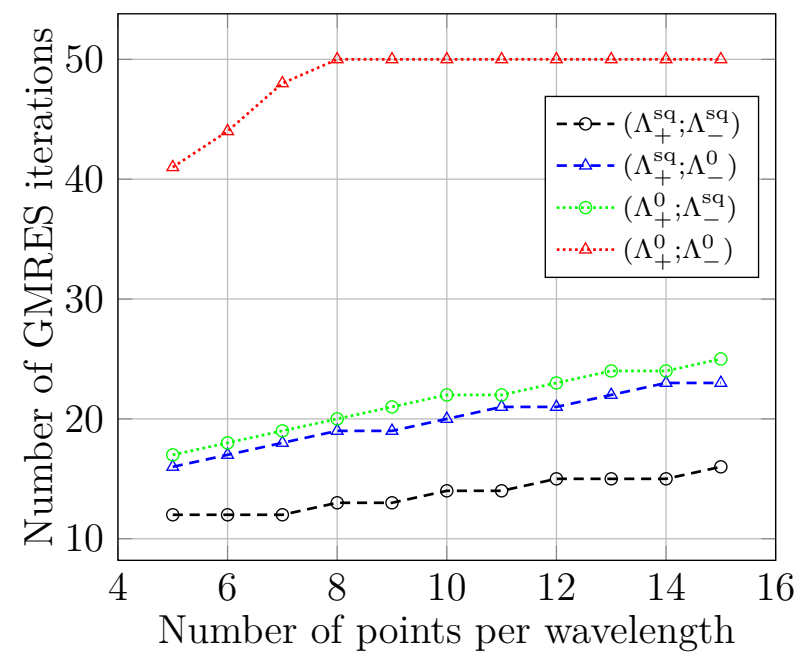

Figure 9: Number of GMRES iterations for the weak FEM-BEM coupling vs. mesh refinement. 
Sphere with tolerance of $10^{-4}$

$k_{-, 0}=19, k_{+}=10, \rho_{0}=5$

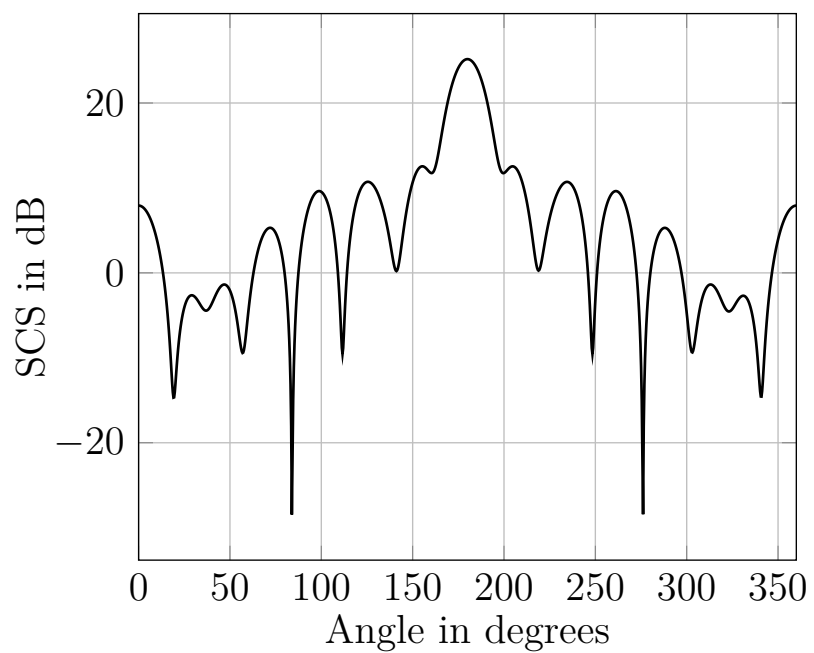

Ellipsoid with tolerance of $10^{-4}$

$$
k_{-, 0}=20, k_{+}=10, \rho_{0}=5
$$

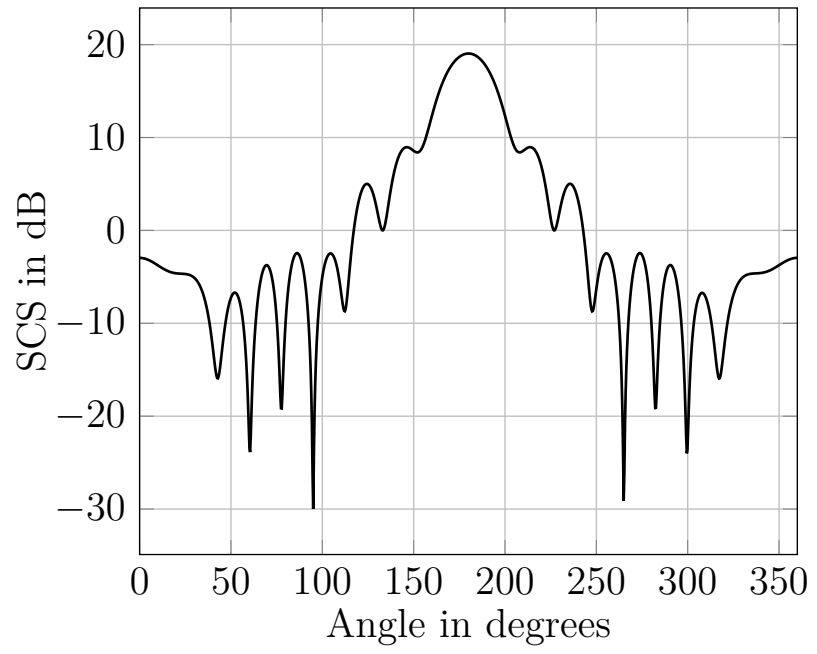

Sphere with several tolerances

$$
k_{-, 0}=19, k_{+}=10, \rho_{0}=5
$$

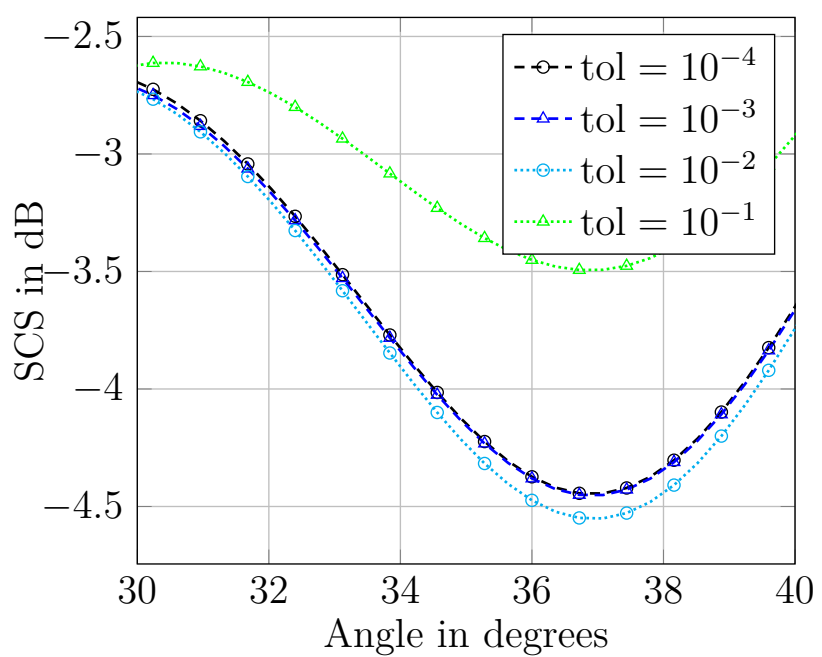

Ellipsoid with several tolerances

$$
k_{-, 0}=20, k_{+}=10, \rho_{0}=5
$$

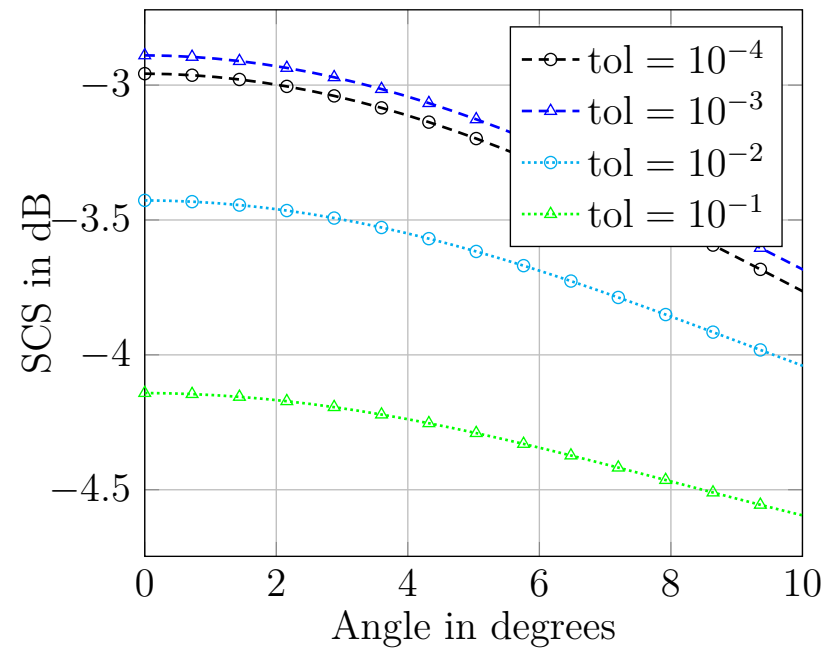

Figure 10: Convergence of the SCS with respect to the GMRES tolerance for the weak FEM-BEM coupling (sphere and ellipsoid) 


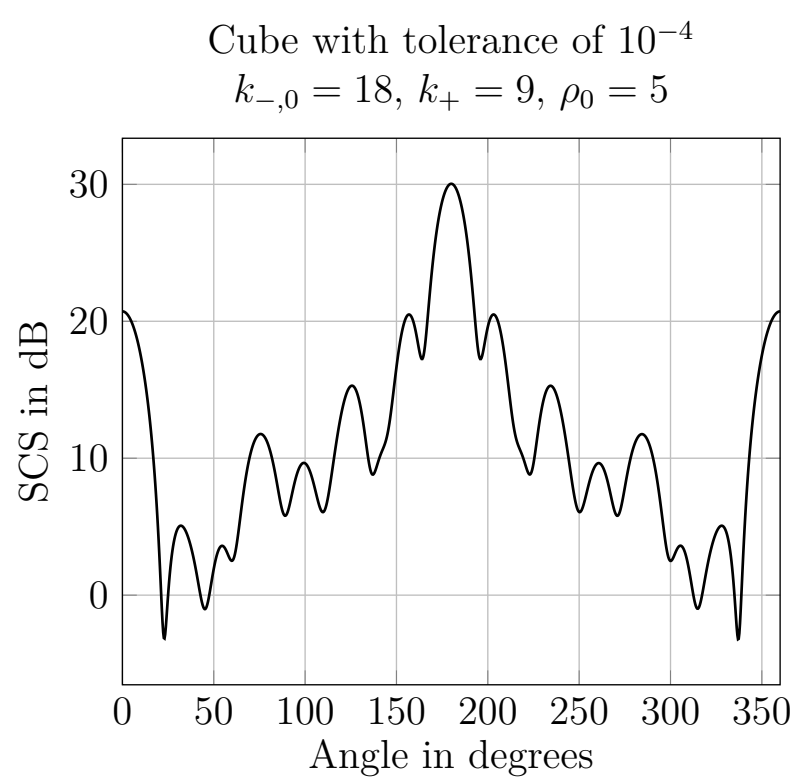

Cube with reentrant corner with tolerance of $10^{-4}$ $k_{-, 0}=17, k_{+}=8, \rho_{0}=5$

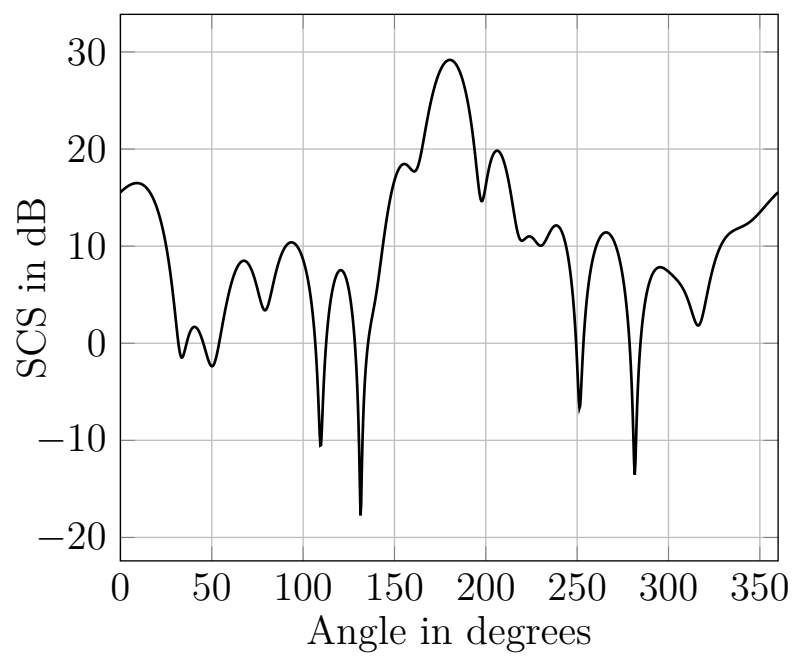

Cube with several tolerances $k_{-, 0}=18, k_{+}=9, \rho_{0}=5$

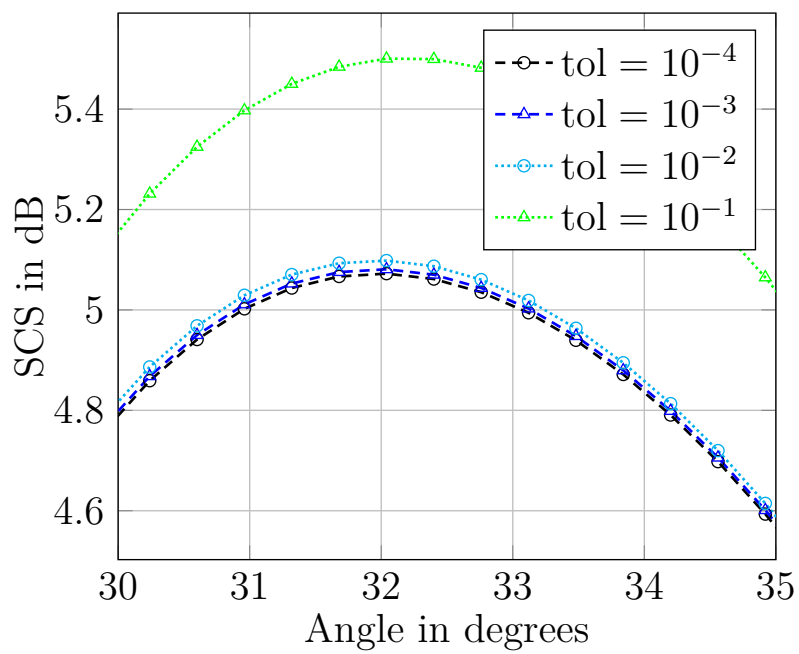

Cube with reentrant corner with several tolerances $k_{-, 0}=17, k_{+}=8, \rho_{0}=5$

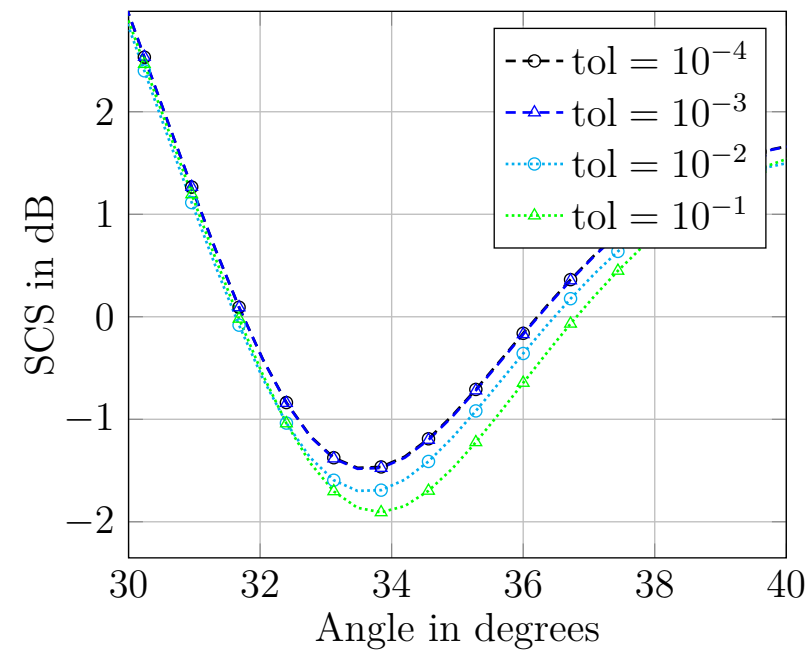

Figure 11: Convergence of the SCS with respect to the GMRES tolerance for the weak FEM-BEM coupling (cube and cube with reentrant corner) 
[5] A. Bendali, Y. Boubendir, and M. B. Fares. A FETI-like domain decomposition method for coupling finite elements and boundary elements in large-size problems of acoustic scattering. Computers \& Structures, 85(9):526-535, 2007.

[6] J.-P. Bérenger. A perfectly matched layer for the absorption of electromagnetic waves. Journal of Computational Physics, 114(2):185-200, 1994.

[7] P. G. Bergmann. The wave equation in a medium with a variable index of refraction. The Journal of the Acoustical Society of America, 17(4):329-333, 1946.

[8] A. Bermúdez, L. Hervella-Nieto, A. Prieto, and R. Rodríguez. Perfectly matched layers for timeharmonic second order elliptic problems. Archives of Computational Methods in Engineering, 17(1):77$107,2010$.

[9] S. Börm. Efficient numerical methods for non-local operators: H2-matrix compression, algorithms and analysis, volume 14. European Mathematical Society, 2010.

[10] Y. Boubendir, X. Antoine, and C. Geuzaine. A quasi-optimal non-overlapping domain decomposition algorithm for the Helmholtz equation. Journal of Computational Physics, 231(2):262-280, 2012.

[11] Y. Boubendir, A. Bendali, and M. B. Fares. Coupling of a non-overlapping domain decomposition method for a nodal finite element method with a boundary element method. International Journal for Numerical Methods in Engineering, 73(11):1624-1650, 2008.

[12] F. Brezzi and C. Johnson. On the coupling of boundary integral and finite element methods. Calcolo, 16(2):189-201, 1979.

[13] B. Caudron. Couplages FEM-BEM faibles et optimisés pour des problèmes de diffraction harmoniques en acoustique et en électromagnétisme. PhD thesis, Université de Lorraine, France, and Université de Liège, Belgique, 2018.

[14] D. Colton and R. Kress. Integral equation methods in scattering theory. SIAM, 2013.

[15] M. Costabel. Symmetric methods for the coupling of finite elements and boundary elements (invited contribution). In Mathematical and Computational Aspects, pages 411-420. Springer, 1987.

[16] A. de La Bourdonnaye. Some formulations coupling finite element and integral equation methods for Helmholtz equation and electromagnetism. Numerische Mathematik, 69(3):257-268, 1995.

[17] B. Després. Méthodes de décomposition de domaine pour les problèmes de propagation d'ondes en régime harmonique. Le théorème de Borg pour l'équation de Hill vectorielle. PhD thesis, 1991.

[18] V. Dolean, P. Jolivet, and F. Nataf. An introduction to domain decomposition methods: algorithms, theory, and parallel implementation. SIAM, 2015.

[19] P. Dular, C. Geuzaine, F. Henrotte, and W. Legros. A general environment for the treatment of discrete problems and its application to the finite element method. IEEE Transactions on Magnetics, 34(5):3395-3398, 1998.

[20] B. Engquist and A. Majda. Absorbing boundary conditions for numerical simulation of waves. Proceedings of the National Academy of Sciences of the United States of America, 74(5):1765-1766, 1977.

[21] O. G. Ernst and M. J. Gander. Why it is difficult to solve Helmholtz problems with classical iterative methods. Numerical Analysis of Multiscale Problems, 83:325-363, 2012.

[22] M.J. Gander, F. Magoulès, and F. Nataf. Optimized Schwarz methods without overlap for the Helmholtz equation. SIAM Journal of Scientific Computing, 24(1):38-60, 2002.

[23] G. N. Gatica. Variational formulations of transmission problems via FEM, BEM and DtN mappings. Computer Methods in Applied Mechanics and Engineering, 182(3-4):341-354, 2000.

[24] L. Greengard and V. Rokhlin. A new version of the fast multipole method for the Laplace equation in three dimensions. Acta Numerica, 6:229-269, 1997.

[25] W. Hackbusch. Hierarchical matrices: algorithms and analysis, volume 49. Springer, 2015.

[26] F. Ihlenburg and I. Babuska. Dispersion analysis and error estimation of Galerkin finite element methods for the Helmholtz equation. International Journal for Numerical Methods in Engineering, 38:3745-3774, 1995.

[27] C. Johnson and J.-C. Nédélec. On the coupling of boundary integral and finite element methods. Mathematics of Computation, 35(152):1063-1079, 1980.

[28] D. Lahaye, J. Tang, and K. Vuik. Modern solvers for Helmholtz problems. Springer, 2017. 
[29] U. Langer and O. Steinbach. Coupled boundary and finite element tearing and interconnecting methods. In Domain Decomposition Methods in Science and Engineering, pages 83-97. 2005.

[30] P.-L. Lions. On the Schwarz alternating method III: A variant for non overlapping subdomains. In Third International Symposium on Domain Decomposition Methods for Partial Differential Equations, pages 20-22. Houston, Texas, 1989.

[31] A. Modave, C. Geuzaine, and X. Antoine. Corner treatments for high-order local absorbing boundary conditions in high-frequency acoustic scattering. Journal of Computational Physics, 401:109029, 2020.

[32] J.-C. Nédélec. Acoustic and electromagnetic equations: integral representations for harmonic problems, volume 144. Springer-Verlag, 2001.

[33] G. Of and O. Steinbach. Is the one-equation coupling of finite and boundary element methods always stable? ZAMM - Journal of Applied Mathematics and Mechanics/Zeitschrift für Angewandte Mathematik und Mechanik, 93(6-7):476-484, 2013.

[34] Y. Saad. Iterative methods for sparse linear systems. SIAM, 2003.

[35] W. Śmigaj, T. Betcke, S. Arridge, J. Phillips, and M. Schweiger. Solving boundary integral problems with BEM++. ACM Transactions on mathematical software, 41(2):6:1-6:40, 2015.

[36] P. Solin, K. Segeth, and I. Dolezel. Higher-order finite element methods. Chapman and Hall/CRC, 2003.

[37] A. Toselli and O. B. Widlund. Domain decomposition methods: algorithms and theory, volume 34. Springer, 2005.

[38] E. Turkel and A. Yefet. Absorbing PML boundary layers for wave-like equations. Applied Numerical Mathematics, 27(4):533-557, 1998.

[39] O. C. Zienkiewicz, D. W. Kelly, and P. Bettess. The coupling of the finite element method and boundary solution procedures. International Journal for Numerical Methods in Engineering, 11(2):355-375, 1977. 